TITLE:

\title{
Hydrodynamic characteristics and related mass-transfer properties in open-channel flows with rectangular embayment zone
}

\author{
$\operatorname{AUTHOR}(\mathrm{S}):$
}

Sanjou, Michio; Nezu, lehisa

\section{CITATION:}

Sanjou, Michio ...[et al]. Hydrodynamic characteristics and related mass-transfer properties in open-channel flows with rectangular embayment zone. Environmental Fluid Mechanics 2013, 13(6): 527-555

\section{ISSUE DATE:}

2013-12

URL:

http://hdl.handle.net/2433/193003

\section{RIGHT:}

The final publication is available at Springer via http://dx.doi.org/10.1007/s10652-0139279-2.; This is not the published version. Please cite only the published version.; この論 文は出版社版でありません。引用の際には出版社版をご確認ご利用ください。 


\title{
HYDRODYNAMIC CHARACTERISTICS AND RELATED MASS TRANSFER PROPERTIES
}

IN OPEN-CHANNEL FLOWS WITH RECTANGULAR SHAPED EMBAYMENT ZONE

\author{
By Michio Sanjou ${ }^{1}$ and Iehisa $\mathrm{Nezu}^{2}$
}

\author{
${ }^{1}$ Associate Professor, Department of Civil Engineering, Kyoto University, Kyoto 615-8540, Japan \\ ${ }^{2}$ Professor, Department of Civil Engineering, Kyoto University, Kyoto 615-8540, Japan
}

Corresponding e-mail :michio.sanjou@water.kuciv.kyoto-u.ac.jp

\begin{abstract}
Consecutive groins and embayment form dead water zones, where sedimentation and congestion of pollutants are often observed. It is thus very important to reveal mass and momentum exchanges between main-channel and side-cavity in river environment and hydraulic engineering. There exists spanwise gradient of streamwise velocity near the junction, and it produces small-scale turbulent vortices owing to shear instability. Furthermore, large-scale horizontal circulations are also generated in the cavity zone. These coherent turbulent structures play significant roles on mass and sediment transfers through a mainstream / embayment boundary. However, the relation between the turbulence and mass transfers is still poorly understood. In the present study, PIV and LIF measurements are conducted in a laboratory flume using a laser light sheets and high-speed CMOS camera, and we compared exchange properties of dye concentration and effects of sedimentation on them among different bed configurations. The both of primary gyre and secondary one are observed in the flat-bed and downward slope conditions, and in contrast, the primary gyre is prevalent in the upward slope condition. It was found that these formations of the horizontal circulations have striking impacts on the mass transfer properties between the mainstream and the side-cavity.
\end{abstract}

Keywords: side-cavity, open-channel flows, mass transfer, turbulence measurements, PIV and LIF 


\section{INTRODUCTION}

Natural rivers are not simple straight streams but include complicated boundaries such as bending and confluence sections. Local scouring and sedimentation are promoted by three-dimensional currents and turbulence structure. Sediment transport is one of significant topics in river engineering. Therefore, various types of spur dykes and groynes have been developed and related studies are conducted intensively by many researchers and engineers, in order to prevent flood disaster and keep proper aquatic ecosystem and water quality.

Photo 1 shows a group of groynes situated in outer curve of Katsura river in Japan, in which a horizontal circulation is typically formed. It is well known that a strong downward flow is generally produced in the outer curve zone due to Prandtl's first kind secondary current, and this induces local scouring and bank erosion. The groynes play important role on navigating flow direction to prevent momentum accumulation of mainstream. So, many researchers are studying basic flow patterns around the groyne, relation between current and scouring and mass transport through the main-channel / embayment boundary. However, three- dimensional current dynamics around the groynes have not be investigated yet, and it thus makes more difficult to accomplish the goals of these studies.

Ercan and Younis (2009) have examined efficiency of the groyne in reducing a bank erosion by using two and three dimensional numerical schemes. In actual rivers, it is of importance to select efficient alternative designs of spur dykes and groynes in river managements. Uijjtewall (2005) has studied effects of different groyne designs which include pearmeable type made by a row of piles, on hydrodynamic characteristics. There are significant differences between streamwise velocities of the mainstream and the cavity zone surrounded by neighboring spur dykes. Of particular significance is that a shear instability related to the velocity difference induces coherent horizontal vortex along the boundary of the mainstream and the cavity. Further, large-scale gyres are formed in the embayment zone in which lots of suspended sediments are trapped conveyed from the main-channel, and local sedimentations are promoted in the cavity as shown in photo 2 . So, it is necessary to reveal the hydrodynamic properties included with turbulence phenomena in order to control sedimentation much reasonably. Akkerman et al.(2004) conducted the sensitivity analysis with a one-dimensional morphodynamic model, and they discussed the several effects on sedimentation and flood water depth after occurrence of groyne damage. Yossef \& Vriend (2010) 
have considered a sediment transport on the basis of laboratory measurements in a mobile-bed flume with consecutive groynes, in which measured data of velocity, free-surface fluctuation and bed profiles are obtained in various hydraulic conditions. Furthermore, they have estimated a bed-form migration reasonably by the PIV technique for both of emergence and submergence cases. They suggested that the double structure of turbulence identified by Nezu \& Nakagawa (1993), i.e., three-dimensional small-scale turbulence and large-scale coherent vortex, is prevalent among and around groynes, and it is closely relevant to the trap and release of the sediment in the dead water zones. In particular, the detached eddies from the groye tip picks up the bottom sediments and they intrude into the main-channels. Such a transport process is mainly by the small-scale turbulence diffusion. Whereas, the large-scale gyre among the groynes conveys the sediment from the main-channel, which corresponds to the advective transports. However, they could not measure vertical profiles of the velocity components, and there still remain uncertainties about three-dimensional hydrodynamic structure. A bed shear stress is closely related to Reynolds stress, and thus it is very important to investigate turbulence distribution for considering sediment transport. Duan et al. (2009) have measured mean flow and turbulence structure near the single spur dyke for both conditions of fixed-flat bed and movable one. They obtained all three components of velocities by using acoustic Doppler anemometer, and explain how is difference of turbulence structures, mean flow patterns included secondary currents and properties of the bed shear stress between the flat and scoured bed conditions. Particularly, it is found that maximum scour depth is observed corresponding to the maximum bed shear stress. Sukhodolov et al. (2004) has conducted field measurements in Elbe River in Germany, and obtained three-dimensional turbulence structure, sediment distribution and scouring property in the embankment region. The turbulence spectra is content well with Kolmogorov's $-5 / 3$ power law, irrespective of depth direction. It was further found that concentration of suspended sediment depends on a distance from a shear layer formed along the mainstream / embayment boundary. Therefore, the turbulence structure is a key role to control the mass and sediment transports in the groyne field.

Recently, permeable groynes are proposed to realize stable bed condition. For example, Kadota \& Suzuki (2010) discussed several effects of the permeability and the scales of the groynes and stone gabion in submerged and emerged flow conditions. They conducted laboratory measurements in open-channel flows with alone groyne model over movable bed, and compared bed configurations varying groyne length and gabion size systematically. There were opposite results for emerged and 
submerged conditions, that is to say, significant local scour emanates near the emerged groyne for the small permeability, and in contrast, sand waves are formed far downstream of the submerged groyne for the same permeability. They concluded that this is due to spatial variation of the shear layer. Tominaga \& Sakaki (2010) conducted ADV measurements around the permeable groynes in a natural river, and they evaluated distributions of bed shear stress which allows us to consider the local scour reasonably. Gu et al.(2011) have pointed out that the aspect ratio of groyne is one of important factors to influence strikingly a transport of suspended sediment around permeable groynes. Further, their measured data suggests that a mass exchange rate of the sediment decreases with rank number of groyne, and keeps constant after the fifth groyne. The local scouring changes dramatically an original bed configuration, hydraulic structures such as spur dyke, groyne and bridge peers would be very unstable. Although, it is well known that these scouring problems are often agued and studied in river streams. It is noted that tsunami and waves running up and down also have striking impacts on the structures. Mioduszewski \& Maeno (2006) have studied experimentally relation between the permeability of the single groyne and scouring characteristics in unsteady waving currents. Particularly, velocity vectors, excess pore pressure and liquefaction are compared for the permeable and impermeable spur dykes.

In these groyne fields, not only sedimentation but also congestion of pollutants is often highlighted. It is thus very important to investigate mass and momentum exchanges between the main-channel and the side-cavity in river environment and hydraulic engineering. The above-mentioned horizontal gyres and coherent turbulence structures play significant roles on these exchange phenomena. Valentine \& Wood (1977) focused on the concentration exchanges between the mainstream and bottom-situated cavity in open-channel flows. In order to evaluate them quantitatively, they proposed a mass transfer flux given under an assumption that the interfacial transfer is proportional to a difference of the concentrations in the mainstream and the cavity. Uijttewaal et al.(2001) measured distribution of dye concentration and pointed out that aspect ratio of side cavity has significant effects on exchange rate of mass between the mainstream and the embayment. They considered dependency of mass transfer coefficient on geometry of groynes, water depth, groyne rank number and bed shape. Note that transfer speed is significantly affected by the aspect ratio. In a small gyre field where the aspect ratio is closely to 1.0, i.e., square horizontal shape, the mass transfer is dominated by the mixing layer formed along the boundary between the mainstream and the dead water zone. As the mixing layer is not penetrated to within the horizontal 
gyre, and thus dye is trapped in the center of the gyre with time proceeding. In contrast, in the large groyne field, coherent shedding vortices are generated from a tip of the upper lank groyne. These are conveyed to the center of the primary gyre (PG), and therefore, the mass exchange is promoted more strikingly than a small gyre field. Furthermore, dye and dissolved materials are caught by the secondary gyre which is shielded from the PG, shedding vortices and the mixing layer. Weitbrecht et al.(2008) have also conducted laboratory measurements, in which distributions of velocity components and dissolved dye were obtained, and examined the relation between the exchange rate and the bed configuration of the cavity. They proposed two methods to determine the mass transfer coefficient were proposed, i.e., 1) by using velocity components, in which the mainstream velocity and cross stream one at the mainstream / embayment boundary are considered, and 2) by using time-decay of concentration with in the embayment, where depth-averaged concentrations were measured by light absorption similar to a typical LIF method. On the basis of measured data, they suggested that dimensionless mass transfer coefficient varies linearly with normalized morphometric groyne field parameter which consists of water depth, width and length of groyne. Constantinescu et al.(2009) have conducted three-dimensional LES to investigate dissolved contaminant exchange through the main-channel / embayment interface. In this their numerical results, the ejection and exchange processes are very non-uniform over the depth. For example, they predicted that many contaminants are moved away from the dead water zone closed to the free-surface. Such a three-dimensionality of mass transfer was pointed out by also Uijttewaal et al. It should be noticed that the predicted results of the mass exchange coefficient are close to the data. Furthermore, it corresponds well to an above mentioned experimental formula proposed by Weitbrecht et al.

However, there remain uncertainties about accurate evaluations of exchange velocity, effects of the bed formation on mass transport and physical modeling of mass exchange in the side-cavity flows. Ettema \& Muste (2004) have targeted on a single spur dyke in rigid bed open-channel flows, in which the horizontal flow field around the spur dyke could be classified into some characteristic zones, considering a flow separation and thalweg alignment, and have examined how these length scales influence hydrodynamic characteristics. A three-dimensional feature of shedding vortices was also explained regarding relationship among development of shedding vortex tube, thelweg disturbance region and flow recovery length to a symmetrical velocity profile. Their study implies that even single spur dyke could produce complicate turbulence structure. Whereas, when more 
than two spur dykes are placed in the streamwise direction, as shown in Photo 1, the dead water zone appears in the neighboring groynes. In the embayment zone, a flow is quite stagnant, and as results, it creates ideal habitat spaces for river environment. So, the side-cavity zones called as "wando" are constructed deliberately in several Japanese rivers under the government project.

Currents structures in the side-cavity channels are closely similar to those observed in the consecutive groyne field. That is to say, there exist shear instability, shedding vortices, horizontal large-scale gyres in the dead water zone. Booij (2004) has conducted laboratory measurements for free-surface flows around the square-shaped side-cavity, and compared the flow patterns among different square sizes. He also considered the development of mixing layer and secondary currents. Side-cavity zones observed in natural rivers are often lost by large amount of sediments after high discharge flow, and dissolved pollutants trapped in the embayment reduce water quality very much. They are emergency and fatal problems to solve as possible as soon. To consider them properly, it is necessary to reveal the three-dimensional flow pattern and related coherent turbulence. Unfortunately, not much is known about these technical and scientific topics.

This paper focuses on the mass transfer properties in open-channel flows with a single side-cavity, and presents quantitative evaluation and physical mechanisms for currents and turbulence structures and dye transfer phenomena. Considering sedimentation in natural embayment zones, we chosen not only a flat bed condition but also slope bed ones as mentioned later. Experimental procedure, measurement methods and hydraulic conditions are described in section 2. PIV measurements and their results are examined in section 3. Time-averaged horizontal flow patterns and turbulence structures are revealed and they are compared among different bed conditions. In the present experiments, flow fields illuminated by both of vertical and horizontal laser light sheets are measured simultaneously by a dual-camera PIV system. This information allows us to understand a relation between the horizontal gyre motions and vertical flow reasonably. In section 4, LIF measurements are conducted to evaluate mass transfer velocity through the boundary between the mainstream and the embayment zone. The measured data suggests that there exists significant trap / release properties of dye transfer in the dead water zone closely related to the turbulence structures. In section 5, conclusions of the present results are described. 


\section{LABORATORY EXPERIMENTS}

\subsection{Laboratory flume and coordinate system}

The experiments were conducted in a $10 \mathrm{~m}$ long, $40 \mathrm{~cm}$ width glass-made tilting flume. The coordinate was chosen that $(x, y, z)$ correspond to the streamwise, vertical and spanwise directions, respectively. The vertical origin, $y=0$, was chosen as the channel bed. The time-averaged velocity components in each direction are defined as $U, V$ and $W$, and the corresponding turbulent fluctuations are $u, v$ and $w$, respectively. Therefore, instantaneous velocity components are indicated as follows; $\widetilde{u}=U+u, \widetilde{v}=V+v$ and $\widetilde{w}=W+w$ for the sreameise, vertical and spanwise directions. The vertical origin $(y=0)$ and spanwise one $(z=0)$ are chosen as the main-channel bottom and the main-channel / embayment boundary, respectively. In the measurement section, $7 \mathrm{~m}$ downstream from the channel entrance, a side-cavity is placed by acrylic plates. The water depth is $H=5.3 \mathrm{~cm}$. The widths of the main-channel and the cavity are $B=30 \mathrm{~cm}$ and $B_{w}=10 \mathrm{~cm}$. The streamwise lenght of the cavity is $L_{w}=30 \mathrm{~cm}$. The water temperature is $20^{\circ} \mathrm{C}$.

\subsection{Measurement method}

The PIV is very useful to evaluate space-time variations of flow patterns, because two or three-dimensional velocity components could be obtained at multi-points simultaneously. There are many applications to open-channel hydraulics (see Nezu \& Sanjou 2011). In the present experiments, the $2 \mathrm{~mm}$ thick laser light sheet (LLS) was generated by $3 \mathrm{~W}$ Ar-ion laser using a cylindrical lens. The illuminated flow images were taken by a CMOS camera $(1000 \times 1000$ pixels $)$ with $100 \mathrm{~Hz}$ frame-rate and $30 \mathrm{~Hz}$ sampling-rate. The sampling duration is 60 seconds for each case. The instantaneous velocity components $(\tilde{u}, \widetilde{w})$ on the horizontal plane were calculated by the PIV algorithm. The spatial resolution is about $0.37 \mathrm{~mm}$ per pixel. The illuminated horizontal plane using tracer particles was taken by a high-speed CMOS camera controlled by a personal computer which is connected with the pulse-signal generator. A trigger signal which has same frequency as data sampling rate $1 / \Delta t(\mathrm{~Hz})$ is transferred to the CMOS camera via the control computer, and a pair of digital images are taken accurately at the frame rate $1 / \Delta \delta(\mathrm{Hz})$ of the CMOS camera. In this study, $1 / \Delta t$ was set $30 \mathrm{~Hz}$ and $1 / \Delta \delta$ was set $100 \mathrm{~Hz}$. The time-variation of the instantaneous velocity vectors was calculated by a PIV algorithm, in which an interrogation size is $29 \times 29$ pixels. When a correlation value between first and second image patterns is less than 0.4 , a local velocity vector 
was judged an invalid vector. Comparison with surrounding velocity vectors is also very efficient to detect suspicious vectors as suggested by Westerweel (1994). In the present study, a local divergence value includes the referred and the surrounding vectors is calculated. The referred velocity vector could be invalid when the corresponding divergence is larger than threshold criteria. This value is quite same as that used by Nezu \& Sanjou (2008), in which profiles of streamwise profile and Reynolds stress measured by PIV in vegetated canopy flows agree well with those obtained by laser Doppler anemometer. After the detection process of the invalid velocity vectors, a spatially-interpolated velocity data was substituted for corresponding invalid velocity vectors. In the "horizontal-LLS measurements", the elevations of the LLS were $y / H=0.19,0.38,0.47,0.57$, 0.75 and 0.94 are chosen for the all cases. We conducted PIV measurements ten times under the same condition for the flat-bottom case in order to examine the repeatability of our experiments. Figs.1 (a) and (b) show lateral profiles of time-averaged streamwise velocity $U(z)$ and horizontal Reynolds stress $-\overline{u w}(z)$ together with their standard deviation $\sigma$ at $x / L_{w}=0.5$ and $y / H=0.47$. It is therefore expected that the present experiments have the measurement repeatability, in which maximum standard deviation is $0.4 \mathrm{~cm} / \mathrm{s}$ and $0.12 \mathrm{~cm}^{2} / \mathrm{s}^{2}$ for time-averaged velocity $U(z)$ and Reynolds strtess $-\overline{u w}(z)$, respectively .

The distribution of dye concentration is measured by the LIF method, in which the sharp-cut filter was put on the lens of the CMOS camera in order to obtain clear concentration image illuminated by the LLS. The instantaneous distribution of the dye concentration was calculated by using brightness values of these LIF image. The dye (Rhodamine-B), the concentration of which is $0.2 \mathrm{mg} / \mathrm{l}$, was dissolved in the cavity. At the initial stage of the measurements, the cavity and the main-channel are separated by a splitter plate. This could be lifted up smoothly along guide rails by the counterweight. It takes 0.1 seconds for the splitter plate to go out of the water body. After remove of the plate, the dye exchange and transfer motions were captured by the CMOS camera. Borg et al. (2001) pointed out that there exists a linear relation between image brightness and dye concentration under the small concentration condition $(\mathrm{C}<0.2 \mathrm{mg} / \mathrm{l})$. Same calibration tests were conducted ten times in the present study. Fig.2(a) shows the relation of already known concentration series and ensemble-mean local pixel intensity in the side cavity. The splitter plate divides the side-cavity from the mainstream as shown in Fig.2(b). These calibration images are taken by the CMOS camera placed over the free-surface. The LLS elevation is $y / H=0.94$. The reflection light power depends largely on a source light power, light path distance, absorption efficiency and so on 
as pointed out by Cowen et al. (2001). The maximum distance of the light path through the dye is $10 \mathrm{~cm}$ which correspond with the cavity's spanwise width $B_{w}$, and thus in order to examine the light decay effects, three calibration curves are compared, for the three different points, i.e., "a", "b" and "c" indicated in Fig.2(b). The corresponding path distance is $2 \mathrm{~cm}, 5 \mathrm{~cm} 9 \mathrm{~cm}$, respectively. The present result suggests that linear relations are obtained approximately for the lower concentration range $(C<0.2 \mathrm{mg} / 1)$. Same tendency could be observed, irrespective of the LLS elevation. However the pixel intensity is scattered a little at $\mathrm{C}=0.2 \mathrm{mg} / 1$. Table 2 shows coefficient of determination $R_{2}$ for linear and quadratic approximations, and this result suggests that differences between them are negligible by small, independent of the path distance. Therefore, we calculated linear calibration formula for all local positions. The standard deviation of the measured pixel intensity with whole 256 levels is indicated in Fig.2(c), which implies that maximum $\sigma$ is 4.4 for the $5 \mathrm{~cm}$ path distance.

\subsection{Hydraulic condition}

Table 1 shows hydraulic condition, in which the bulk-mean velocity is $U_{m}=12 \mathrm{~cm} / \mathrm{s}$, Froude number is $F r=0.166$ and Reynolds number is $R e=6360$. We chosen several kinds of bed configurations in the cavity as shown in Fig.3. Various sedimentation patterns are observed in real system depending on current velocity, water depth and sediment properties. For example, large particles such as bed load sediment tend to be caught in the downstream-side within the cavity, and in contrast, it is possible that small ones such as suspended sediment are transferred by the large-scale gyre of the cavity and trapped in the upstream-side with in the cavity. After natural flood and flashing discharge operation of artificial dams, the cavity zones are sometimes filled with sediments as shown in Photo 2. So, we deal with the following model cases in which bed elevation varies in the streamwise direction. $S$ is a parameter to indicate the bed configuration. Negative sign of $S$ means downward incline as shown in Fig.1 (b), and positive one means upward incline as shown in Fig.3(c). $S=0$ means the flat bed condition. The absolute value of $S$ means the inclination of the bed. In the present study, maximum heights of bottom slope are $2 \mathrm{~cm}$ and $5 \mathrm{~cm}$ for the mild and steep conditions, respectively.

\section{HYDRODYNAMIC CHARACTERISTICS OF FLOW PATTERN AND TURBULENCE STRUCTURE}




\subsection{Horizontal velocity components in time-averaged field}

Fig.4 shows time-averaged horizontal velocity vectors $(U, W)$ at $y / H=0.46$ and 0.94 . At the near free-surface, $y / H=0.94$, two kinds of large scale gyres with counter rotations are observed in the flat bottom condition, $S=0$ and the downward-slope condition, $-1 / 6$. Whereas, single gyre structure appears and it covers a whole region of the canopy in the upward-slope condition, $S=1 / 6$. The primary gyre $(\mathrm{PG})$ is a horizontal circulation induced directly by the main-stream momentum, and it was observed, irrespective of the elevation and the geometry of the main-channel bottom. The secondary gyre (SG) seems to be generated by the PG. In the $S=1 / 6$, the contact area between the main stream and the cavity zone decreases downstream, and the inflow from the mainstream toward the side cavity becomes smaller compared with those observed in the $S=0$ and $-1 / 6$. Thus, the position of PG is shifted toward upstream-side of the embayment, and the sufficient space could be not kept to generate the SG in the $S=1 / 6$. At the mid-depth layer, $y / H=0.47$, the PG and the SG are observed in the same manner as those of $y / H=0.94$ in the flat-bottom condition. In contrast, the only PG is formed in the mid-depth layer in the slope-bottom conditions.

Fig.5(a) shows longitudinal variations of time-averaged spanwise velocity component $W$ at the different elevations, i.e., $y / H=0.18,0.47$ and 0.94 in the case of the flat bed condition, $S=0$. The spanwise position is the junction, i.e., $z=0$ for all elevations. It is found that inward flows to the embayment and outward flows from that are prevalent near the upstream and downstream zones of the side cavity, respectively. It should be noticed that the inward current is largest at mid water-depth, $y / H=0.47$. This flow is smaller near the free-surface, $y / H=0.94$. In contrast the outward current emanates near the flume bottom, $y / H=0.19$. This result implies that the formations of longitudinal secondary currents are may be varied corresponding to the horizontal flow field. Total of three elevated data has the strong outward current form the embayment, $W>0$, at a mid-length position, $x / B_{w}=1.5$, and the peak inward current, $W<0$, at the downstream side, $x / L=2.5$. It corresponds well to the current's direction of the PG. Fig.5 (b) compares quantitatively among the different bed configurations. The larger outward current at $x / B_{w}=0.3$ and inward current at $x / B_{w}=2.7$ are observed in the case of $S=-1 / 6$ than in the flat bed case of $S=0$. Whereas, in the case of $S=-1 / 6$, significant inrushes happen significantly at the mid-length section of $x / B_{w}=1.5$ due to the shifted PG toward the upstream-side indicated by Fig.4. Further, strong 
outward flows are also generated in the downstream region of the cavity. They are may be a part of flows uncaught by the PG. These results suggest that the bed configuration within the embayment zone influences strikingly the momentum properties through the boundary between the mainstream and the dead water zone.

In the case of the downward slope condition, the contact area between the main-channel and the embayment reduces linearly upstream, and it causes that momentum transfer from the mainstream to the cavity is likely to be concentrated on the downstream-side. This fact is well recognized in Fig.5 (b). These inward currents vary to the counter flow near the side-wall of the embayment, and thereafter, they degenerates owing to viscous friction at the midstream section of $x / B_{w}=1.5$. Satisfying the continuity equation produces positive spanwise flow accompanied by a formation of SG in the upstream corner, $\left(0<x / B_{w}<1.0\right.$ and $\left.-1.0<z / B_{w}<0\right)$. In the case of the upward slope condition, the contact area increases linearly upstream, and the longitudinal position of lateral momentum transfer is shifted toward the upstream. Further, the starting position of the counter flow is also observed in the upstream side compared to the downward slope condition. This tendency keeps the counter flow near the upstream edge of the embayment, and as the result, no corner space to generate the SG is found.

\subsection{Relation between large-scale gyre and bed configuration}

Figs. 6 (a) and (b) show the distributions of time-averaged vorticity with vertical axis, $\Omega \equiv \partial U / \partial z-\partial W / \partial x$ and delta value $\Delta$ at $y / H=0.94$, for $S=-1 / 6,0$ and $1 / 6$, respectively. Chong and Perry (1990) considered the Eigenvalue $\sigma \square$ of the velocity shear tensor $\left(\partial \tilde{u}_{i} / \partial x_{i}\right)$ in shear layers. The Eigenvalue equation of $2 \mathrm{D}$ shear flow is given by

$$
\sigma^{2}-P \sigma+Q=0
$$

in which

$$
P \equiv \frac{\partial \tilde{u}_{i}}{\partial x_{i}}=0 \quad \text { and } Q \equiv \frac{1}{2}\left(\left(\frac{\partial \tilde{u}_{i}}{\partial x_{i}}\right)^{2}-\frac{\partial \tilde{u}_{i}}{\partial x_{i}} \frac{\partial \tilde{u}_{j}}{\partial x_{j}}\right)
$$




$$
\Delta \equiv P^{2}-4 Q
$$

where $\tilde{u}_{i}=$ instantaneous velocity components with $i=1$ and 2 herein. Complex Eigenvalues occur if the discriminant $\Delta$ is negative. Chong and Perry assumed that $\Delta<0$ corresponds to the existence of a vortex core, and verified the validity of such a vortex detection, referred to as the delta method in shear layers. Positive and negative values of the vorticity mean anti-clockwise and clockwise rotations, respectively. It should be noticed that even if the fluid does not rotate, large vorticity appears in the shear layer, where spatial gradient of the velocity components is dominant. Therefore, in the present study, not only the vorticity but also the delta value is used to detect gyre regions and to measure the corresponding areas.

In the flat bed condition, $S=0$, positive distribution of $\Omega$ is observed along the junction, in which the streamwise velocity shear $\partial U / \partial z$ is very large and furthermore small-scale shedding vortices are generated periodically by the shear instability. It is noted that the positive and negative regions appears corresponding to the PG and the SG as shown in Fig.4. It is found that the delta value becomes negative in these regions. In $S=-1 / 6$, the distributions of the vorticity and delta seem to be similar to those of the flat condition. The positive voriticity region corresponding to the PG shifts toward the downstream-side compared to the flat-bottom condition. This property is also observed in the distribution of the delta. This is because the PG is transported toward the downstream-side in the cavity due to the effect of the bed configuration. In contrast, in $S=1 / 6$, the positive vortisity and the negative delta are dominant in the cavity. These properties correspond well with the horizontal distributions of velocity vectors as shown in Fig.4. In the upstream side of the cavity, the negative vorticity and the negative delta appear corresponding to the SG which becomes very small due to the bed configuration effect.

The total area of the PG was calculated by summing the local square, in which the positive vorticity and negative delta appear. In the same way, the total area of SG was calculated by the local square, where negative vorticity and negative delta exist. Fig.7 shows areas of the PG and the SG normalized by a total cavity area at each elevation. The PG is more prevail than the SG in whole depth region, irrespective of the bed configuration type. In the $S=0$ and $-1 / 6$, the area of PG is comparable with that of SG near the free surface. In contrast, in the $S=1 / 6$, the area of PG is larger and that of SG is smaller than those observed in $S=0,-1 / 6$. In the flat condition of $S=0$, a 
vertical variation of the PG area is comparatively small, and in contrast, one of the SG has a blunt peak in the mid-depth zone. Therefore, we can hardly say with any finality that the large-scale gyre structure is horizontally two-dimensional.

\subsection{Distribution of Reynolds stress}

Fig. 8 shows horizontal distributions of Reynolds stress $-\overline{u w}$ at $y / H=0.47$ and 0.94 . The large and positive Reynolds stress zone is formed along the junction between the mainstream and the cavity. The shear instability induces such a strong turbulence layer irrespectively of the bed configuration. It is found that Reynolds stress becomes largest in the downward slope condition, $S=-1 / 6$. A peak Reynolds stress appears at $x / B_{w}=1.0$ in the down slope condition, $S=-1 / 6$. In contrast, all most constant value is observed except the upstream and downstream walls in the cavity. The Reynolds stress of the downward slope condition $(S=-1 / 6)$ is twice as large as those of the flat $(S=0)$ and the upward slope conditions ( $S=1 / 6)$.

In the case of $S=-1 / 6$, strong ejection and sweep events are observed around the mid-stream region, $x / B_{w}=1.0$ to 1.5 . A generation term of Reynolds stress $-\overline{u w}, G_{-\bar{w}}$ is given in the following way.

$$
G_{-\bar{w}} \equiv \overline{u w} \frac{\partial W}{\partial x}+\overline{w w} \frac{\partial U}{\partial z}+\overline{u w}\left(\frac{\partial U}{\partial x}+\frac{\partial W}{\partial z}\right)
$$

Particularly, the second term is the most significant in the horizontal mixing layer, in which the gradient of streamwise velocity component is formed in the lateral direction. The coherent motions such as ejection and sweeps induces large turbulence intensity for the spanwise velocity fluctuation, $\overline{w w}$, and it results in Reynolds stress becomes large due to the increased generation term . In the case of $S=1 / 6$, a counter flow area $(U<0)$ is smaller than one observed in the other cases, because the SG is very small. Consequently, small Reynolds stress is produced through the reduced velocity shear $\partial U / \partial z$.

In the flat-bottom condition, owing to the generation of bed turbulence, the larger Reynolds stress is observed in the mid-depth layer than in the free-surface. Through the passing of the shedding vortices accompanied with the upward currents, large turbulence structure near the bottom layer is transported toward the free-surface. The turbulence is diffused during this vertical transport process, 
and thus, it becomes smaller nearer the free-surface. The Reynolds stress in the free-surface is obviously larger in the downward-slope bed condition than in the flat one. This may be because the distance between the flume bottom and the free-surface is very small in the upward-side of the embayment, and then there, larger turbulence is generated and convected downstream. The Reynolds stress of the upward-slope bed condition, $S=1 / 6$, is reduced than that of the flat condition. Because the PG is situated in the upstream-side in the embayment in this bed condition, the velocity shear and turbulence production become smaller than other bed conditions.

\subsection{Instantaneous velocity properties}

Fig.9(a) shows time-series of the distributions of instantaneous Reynolds stress - $u w$ and descriptions of horizontal velocity components $(\widetilde{u}, \widetilde{w})$ every 1 s for $S=0$. The elevation is $y / H=0.94$. At $t=0 \mathrm{~s}$, the circle (A) indicates the coherent structure of fluid parcel which is transported strongly toward the mainstream. This is called as sweep motion in the present study. The shedding vortex follows the sweep, in which the fluid parcel intrudes into the cavity accompanied by the strong positive Reynolds stress. This is called as ejection. These coherent motions are convected downstream at $t=1 \mathrm{~s}$. A new sweep motion appear as indicated by the circle (B). At $t=2 \mathrm{~s}$, it is found that a large amount of fluid is transported toward the cavity zone by the sweep (B). Furthermore, a shedding vortex is generated together with positive Reynolds stress. This result suggests that mass transfer process between the mainstream and the cavity is promoted significantly by such coherent structures.

Figs.9 (b) and (c) show examples of the instantaneous velocity fields for $S=-1 / 6$ and 1/6. Flow observation of motion picture suggests that the shedding vortex is generated cyclically with constant time span at the upstream edge of the side cavity the generation periodicity is almost same, independent on the bed configuration. In $S=-1 / 6$, the ejection and sweep motions are formed as indicated by (A) and (B) at $t=0 \mathrm{~s}$. Particularly, the sweep (B) is accompanied by the shedding vortex, and in contrast, there exists the vortex structure around the ejection. A new ejection (C) appears at $t=2 \mathrm{~s}$ and contributes significantly to excrete mass trapped in the cavity. It is inferred that the ejection events are generated closely related to the PG motion. In $S=-1 / 6$, there is some possibility of that momentum is concentrated in the mid stream zone of the cavity due to shallow water depth, and this results in the stronger ejections are produced than those of $S=0$. It is found that the PG is 
also visualized even in the instantaneous velocity field. In $S=1 / 6$, the strong sweep are formed at $t=1 \mathrm{~s}$. However, the shedding vortex could not be detected in the same manner as $S=0$ and $-1 / 6$. This result corresponds well to the finding that Reynolds stress is smaller in the $S=1 / 6$ than the $S=0$ and $-1 / 6$ as shown in Fig. 8 .

It is found that the bed configuration has striking impacts on turbulence production in the cavity. It should be noticed in $S=-1 / 6$ in which largest Reynolds stress is produced in the junction, that two kinds of convection patterns are formed, i.e., i) shedding vortices are transported downstream along the main-channel / the cavity boundary and most of them inrush into the side-cavity near the downstream edge, ii) shedding vortices are pushed away toward the main-channel by the strong ejection event produced within the cavity. These significant patterns contribute to inward and outward transfer processes, respectively, and they appear alternatively. These notable coherent motions result in effective mass exchange between the mainstream and dead water zone.

In previous results, horizontal currents are mainly focused, and thus, there remain many uncertainties about vertical velocity component. Particularly, it is very significant to reveal how horizontal vortices and gyres induce upward and downward flows. Therefore, horizontal and vertical LLSs are projected simultaneously, and these illuminated planes are taken by dual CMOS cameras. Fig.10 shows examples of instantaneous velocity vectors in the horizontal and vertical planes. The positions of these planes are $y / H=0.94$. (near free-surface) and $z / B=-0.2$ (near the junction between the main-channel and the cavity). The white broken line of horizontal views and the red one of vertical views indicate the positions of vertical and horizontal illuminated planes, respectively. The contours mean distributions of instantaneous Reynolds stress $-u w$ and vertical velcotiy $\widetilde{v}$ for the horizontal and vertical views, respectively.

In the $S=0$, red circle indicates large Reynolds stress observed near the junction in the cavity. It is inferred that the shedding vortex produces locally the large Reynolds stress distribution. Further, the Reynolds stress seems to intrude toward the cavity. It is found by comparison of the horizontal and vertical views that upward currents appear in the intruding region of large Reynolds stress. The downward current follows the upward one, and it is thus suggested that strong 3-D structure is formed near the junction, when the shedding vortices enter the cavity. In the $S=-1 / 6$, a similar 3-D structure is observed in the same manner as one of $S=0$. Particularly, after the transfer of Reynolds stress toward the cavity, it is accompanied by the downward current in the downstream side of the cavity. In the $S=1 / 6$, upward currents are dominant in the whole area of the vertical plane. Of 
particular significance is that larger upward flows are generated than the surrounding positions at the inrushing time of sweep motion.

\subsection{Correlation properties of horizontal motions}

A correlation analysis allows us to understand how far high-correlated fluid parcel is traveling downstream. Space-time correlation function for the spanwise velocity component is given by

$$
C_{u u}=\frac{\overline{\widetilde{w}\left(x_{0}, y_{0}, z_{0}, t_{0}\right) \widetilde{w}\left(x_{0}+\Delta x, y_{0}+\Delta y, z_{0}+\Delta z, t_{0}+\tau\right)}}{w^{\prime}\left(x_{0}, y_{0}, z_{0}, t_{0}\right) w^{\prime}\left(x_{0}+\Delta x, y_{0}+\Delta y, z_{0}+\Delta z, t_{0}+\tau\right)}
$$

, in which $w^{\prime}$ is the turbulence intensity for the spanwise velocity component and $\tau$ is a time delay . The suffix " 0 " means a referred position situated at $\left(x_{0}, y_{0}, z_{0}\right)=\left(0.7 B_{w}, 0.94 H, 0\right)$. Fig.11

indicates time-series of $C_{w w}$ at $y / H=0.94$. It is recognized for all cases that high correlation region between the refereed and movable positions is convected by the mainstream. In the results of $\tau=0$, trajectory lines are depicted connecting maximum correlation positions of each time-decay. In the case of $S=-1 / 6$ and 0 , this line crosses the boundary near the downward-side of the cavity. In contrast, it is noted that the high-correlation parcel enters the embayment in the mid-stream zone $\left(x / B_{w} \approx 1.5\right)$ of the boundary, because the PG is shifted to the upstream-side for $S=1 / 6$. These notable differences among the bed configurations give striking impacts on the mass transfers through the main-channel / cavity interface.

Fig.12 shows the longitudinal variation of $C_{w w}$ at the different time-delay. As increase of the time-delay, first peak value of $C_{w w}$ is typically reduced. Note that $C_{w w}$ decreases most remarkably in $S=5$. Two physical reasons could be considered, i.e., i) $\widetilde{w}$ is reduced much more closer to the downsream-end of the cavity due to the increase of bed elevation and ii) the shedding vortex and related coherent fluid parcel are shifted into the cavity zone.

Second peaks are observed in $S=-1 / 6$ and 0 , and thus, traveling distance could be evaluated during constant time. This information gives us the periodicity of the shedding vortices.

\section{DYE TRANSFER POROPERTIES BETWEEN MAINSTREAM AND EMBAYMENT}

\subsection{Comparison of instantaneous concentration patterns among different bed slope conditions}


Fig.13 shows instantaneous brightness of dye illuminated by the LLS after 20 seconds from remove of the splitter plate, in which a target elevation is $y / H=0.94$. High brightness level corresponds to the large concentration of dye. At the time of partition remove, bright zone is observed only within the side cavity, and it is found that mass exchanges beneath the junction is promoted much more with the time proceeding. In the case of $S=-1 / 6$, the dye distribution corresponds well to two kinds of formations of PG and SG. That is to say, the dye disappears in the PG zone due to the intense mass exchange, and in contrast, it makes a long stay in the SG zone. The same tendency could be in the case of $S=0$, in which it seems that the dye volume is a little more than that $S=-1 / 6$. In the case of $S=1 / 6$, a high concentration region with elliptical shape is organized from the upstream region to the midstream one in the cavity. This elliptical zone is out of contact with three sidewalls within the cavity, and it exists in the center of the large-scale PG shown in Fig.4. These results imply that during a transition of $S$ from positive toward negative, the dye retention is promoted much more within the SG, and whereas, outflow process becomes more dominant rather than the retention within PG. Consequently, time-averaged flow patterns such as the PG and the SG have significant relation with the mass transfer between the main-channel and the dead water zone.

Fig.14 shows time-series of concentration distribution in the case of $S=-1 / 6$, every one second. Instable patterns are observed indicated by circles, (A) and (B) corresponding to the shedding vortices at $t=4.0 \mathrm{~s}$. A low concentration zone is situated at the downstream-side of the embayment as indicated by circle (C). This is caused by momentum transfer from the main-channel toward the cavity, and relevant to the inward flows induced by the PG. The circles, (A) and (B) are transferred downstream by the streamwise current. A vortex (A) is developing during convection, and related strong outward flow toward the main-channel is generated at $t=5.0 \mathrm{~s}$. It seems that circle (C) is shifted to the side-wall of the cavity. At $t=6.0 \mathrm{~s}$, circle "B" forming locally a low concentration zone is transferred into the cavity, and another poor concentration zone (C) is returning back upstream. The bulge structure toward the main-channel is also convected downstream and a shedding vortex pattern (D) is newly born in the upstream-side of the embayment. At $t=7.0 \mathrm{~s}$, the low concentration parcel (C) is transferred to the main-channel. It is found that the low concentration parcel goes round the PG core after the inrush toward the embayment.

Of particular significance is that there exist two kinds of traveling motions of the shedding vortex. One invades into the side cavity corresponding concentration parcels (B), (C) and (D), and another is pushed away toward the mainstream corresponding (A). A long-time observation allows us to 
understand these traveling patterns appear alternatively. A sequential process of inward and outward actions results in most effective mass exchange as mentioned later.

\subsection{Evaluation of mass transfer coefficient}

Valentine \& Wood (1977) have focused on the mass exchange phenomena between a mainstream and trench in channel bed, and they proposed a mass transfer coefficient $k$ in a following form. In their idea, mass exchange flux per unit depth and streamwise length could be given as $k U_{b}\left(c_{m}-c_{c}\right)$ under assumption that the mass exchange is proportional to concentration difference between the mainstream and the bottom trench. The mass flux is calculated by

$$
q=k U_{b} L\left(c_{m}-c_{c}\right)
$$

, in which $U_{b}$ is the time-averaged streamwise velocity at the boundary of between the main-channel and the cavity zone. $c_{m}$ and $c_{c}$ are concentration in the main-channel and the cavity, respectively. $L$ is a length of the junction. When the mass flux is represented by $q=\partial\left(\widetilde{c} L B_{w}\right) / \partial t$, a following form is obtained.

$$
\frac{\partial \widetilde{c}}{\partial t}=\left(\frac{k U_{b}}{B_{w}}\right)\left(c_{m}-c_{c}\right)
$$

Therefore, the time-variation of concentration is calculated in the Eq.(8),

$$
c_{c}=D_{0} e^{-\frac{t}{T}}
$$

in which $D_{0}$ is integral constant and $T$ is a retention time of the concentration in the cavity zone. Fig.15(a) shows an example of time-variation of concentration within the cavity normalized by initial concentration $C_{0}$, in which $\log c_{C}$ is proportional to time between $t=0$ and 60 to $70 \mathrm{~s}$, that is to say, the first-order reduction $t_{r}$ is about $65 \mathrm{~s}$. This result implies that $D_{0}$ and $T$ are surely constant values, and therefore a following relation is obtained.

$$
k=-\frac{B_{w}}{U_{j}} \cdot \frac{1}{T}
$$

Eq.(9)and time-series data of concentration allow us to evaluate $k$.

Assuming that vertical transfer is much smaller than horizontal one, the mass transfer coefficient 
$k_{V}$ for the whole volume cavity could be also evaluated in the following form by a integral of each elevated transfer coefficient $k(y)$.

$$
k_{V}=\frac{1}{\sum_{y} L_{w}(y)} \sum_{y}\left(k(y) \times L_{w, y}\right)
$$

Before, evaluating the mass transfer velocity, we have to consider how much is hydraulic impacts of removal action of the splitter plate on turbulence and related mass exchange properties. In particular, a significant key factor is a time-scale from stagnant status to the equilibrium situation. Fig.15(b) shows time-series of instantaneous velocity components $\tilde{u}$ and $\widetilde{w}$ on the junction, $x / B_{w}=1.5$, $y / H=0.47$ and $z=0$. It is found that there exists a remarkable initial time-stage in $t<3.0$ second, and thereafter, velocity signals look like varying to the equilibrium situation. Therefore, it results in the spin-up time-scale $t_{s}$ is about 3.0 second. The ratio of $t_{s}$ to the residence time $t_{r}$ is less than $5 \%$ order, and thus, this fact suggests that measurement errors caused by the removal action of the splitter plate could be neglected.

Fig. 16 shows the relation of the volume-averaged transfer coefficient and the bed configuration. It is found that the mass transfer coefficient for the whole volume increases with decrease of bed slope $S$, and this suggests that downward slope condition promotes most intensively mass exchanges between the main-channel and the side cavity zone.

Weitbrecht et al. (2008) proposed non-dimensional parameter, $R_{D} \equiv B_{w} L_{w} /\left[H\left(B_{w}+L_{w}\right)\right]$, considering that formation of the shedding vortex is influenced by horizontal geometry and water depth of the dead water zone. They reported there exists linear relationship between the measured transfer coefficient and $R_{D}$. The predicted value of $k$ corresponding to the present experiments ( $R_{D}=1.4$ ), is 0.01 , and this is very close to the measured range $k=0.010$ to 0.014 as shown in Fig.16. However, their linear model could not consider the effects of bed configuration. As they asserted the mixing layer play significant roles on the mass transfer, and the turbulence statistical values such as Reynolds stress should be included in prediction formula for the mass transfer velocity.

\subsection{Relation between the mass transport and Reynolds stress}

When a dissolved material is transferred with self-diffusion, a time-variation of concentration is indicated by the following form using the mass transfer velocity $k_{L}$. 


$$
\frac{\partial C}{\partial t}=\frac{A}{V} k_{L} \Delta \mathrm{C}
$$

$A$ is a area through which mass flux pass and $V$ is the water volume. Assuming that $A$ is a contact area of the main-channel and the cavity zone leads to the relation between $k$ and $k_{L}$.

$$
k_{L}=\frac{k U_{b}}{B_{m}} \times \frac{V}{A}=k U_{b}
$$

It is expected that the coherent structure and shedding vortices contribute significantly to the mass exchange through the mainstream / embayment boundary. These turbulence events are relevant to the production of horizontal Reynolds stress, $-\overline{u w}$. Both of the inward action of high-speed fluid in the main-channel called "sweep" and the outward action of low-speed fluid within the embayment called "ejection" produce instantaneous positive Reynolds stress much intensively. In addition to such intermittent events, shedding vortices appear periodically at the upstream corner of the embayment corresponding to the positive Reynolds stress. Therefore, it is inferred that the horizontal Reynolds stress is closely relevant to the mass transfer velocity. Fig.17 compares a square root of the Reynolds stress space averaged along the boundary near the free-surface $\sqrt{-\overline{u w}}$ with the transfer velocity $k_{L}$ at the same hydraulic condition, which are same dimension, i.e, a velocity scale. This result implies that the transfer velocity becomes larger as the Reynolds stress increases, irrespectively of the bed slope condition, and the large-scale turbulence promotes effectively the mass exchange. Although, it includes some scatters, this is owing to existence of other significant factors related to the mass transfer. That is to say, the formation of large-scale gyres within the embayment indicated in Fig.4 and three-dimensionality of inward / outward currents as shown in Fig.10 are also important actions defining the transfer property. When we conduct physical modeling of them, a proper universal formula will be possible for the mass transfer prediction.

The production of Reynolds stress is closely relevant to the velocity shear $\partial U / \partial z \cdot-\overline{u w}$ is given by using the Prandtl's mixing length model in the following way.

$$
-\overline{u w} \equiv l^{2}\left|\frac{\partial U}{\partial z}\right| \frac{\partial U}{\partial z}
$$


Eq.(13) allows us consider the possibility of the proportionality, $k_{L} \propto l|\partial U / \partial z|_{z=0}$. Here, we chosen the half-width obtained from the lateral profile of $-\overline{u w}$ in the boundary as $l$. Fig.18 shows the relation of the measured $k_{L}$ and $l|\partial U / \partial z|_{z=0}$, in which $l|\partial U / \partial z|_{z=0}$ is averaged in the streamwise direction. This result suggests explicitly that there exists linear relationship and the transfer velocity could be linked even to the velocity shear very well.

\section{PHENOMENOLOGICAL MODEL}

The above-mentioned present results let us know that large-scale gyre structure and mixing-layer formation depend significantly on the bed configuration. Fig.19 shows a phenomenological flow model which focuses on current structure and mass transfer property. In the downward-slope condition $(\mathrm{S}<0)$, two kinds of horizontal gyres, i.e., $\mathrm{PG}$ and $\mathrm{SG}$, are produced in the same fashion as the flat-bottom condition. Counter flows formed in the boundary region of the PG / SG reduce the streamwise velocity component in the shear layer within the cavity, and it thus yields large velocity difference between the mainstream / the dead water zone together with strong shear instability and related intensive shedding vortices. The formation of the large velocity shear results in generation of high Reynolds stress and effective mass transfer. Furthermore, notable coherent events, that is to say, ejection and sweep motions are observed in the midstream region which corresponds with the PG / SG boundary. Unfortunately these generation mechanism has not been known yet. It is possible for the shedding vortex entering the cavity to be trigger to induce coherent events. These ejection and sweep events also promote significantly the efficient mass exchanges. A part of mass dissolved in the cavity is captured by the SG, and in contrast, the PG contributes to the mass transfer through the mainstream / cavity boundary.

In contrast, in the upward-slope condition $(\mathrm{S}>0)$, the position of $\mathrm{PG}$ core is moved upstream and then the SG zone is much reduced. Consequently, the streamwise velocity component near the junction within the cavity has a positive sign even in the upstream-side, and this results in that the velcoity shear $\partial U / \partial z$ decreases remarkably. The generated Reynolds stress becomes smaller, and thus the exchange property is much poorer than that of the downward-slope condition. It should be noticed that the PG catches the mass situated in the cavity. This is quite different from the property observed in the downward-slope bed. 


\section{CONCLUSIONS}

In the present study, the open-channel flows with dead water zone were highlighted, and the effects of sedimentation on horizontal circulation structure and related mass-exchanges were considered on the basis of the measured data obtained by the PIV experiments. The main findings obtained are as follows:

1) In the time-averaged horizontal velocity fields, the large-scale circulations are formulated, irrespective of embayment-bed gradient. Particularly, there exist two kinds of gyres in the flat and downward-slope bed conditions, that is to say, one is the primary gyre (PG) caused directly by the streamwise momentum in the main-channel, and another is the secondary gyre (SG) which is induced by the PG. The horizontal core positions of these gyres are almost constant in the vertical direction. Note that the PG is prevalent in the cavity zone in the upward slope condition, and the PG core is shifted toward upstream-side compared to those of the other conditions.

It is found form the distribution of $W$ in the flat-bed condition that strong outward flows toward the mainstream are generated in the mid-depth zone, in contrast those are small in the bed and free-surface zones. This suggests that after long time-averaging, inward current and outward one are observed in the near bed / free-surface zones and in the mid-depth zone, respectively. Focusing on the free-surface region, the outward currents in the upstream-side and the inward currents formed in the downstream-side become more remarkable in the downward-slope bed than in the flat one. Whereas, there are strong inward flows in center of the boundary between the mainstream and the side-cavity in the upward slope condition. It is therefore found that the time-averaged velocity properties are quite different from those observed in the flat and the downward-slope bed conditions.

2) High Reynolds stress parcels are convected around the mainstream / embayment boundary, corresponding to the coherent shedding vortices due to the shear instability. Comparing the instantaneous velocity vectors in the vertical and horizontal planes, we revealed that the vertical upward currents are produced at the inward position of the high-speed fluid with large Reynolds stress. It is found that the downward currents are generated after the inward high-speed flows collide with the downstream-side wall in the embayment. Most previous studies assumed that these 
flow fields are horizontally two-dimensional. However, the present results imply that there exist significant three-dimensional turbulence structures in and around the side-cavity fields.

3) The positive Reynolds stress corresponding to the shedding vortices is observed in the boundary in all hydraulic cases. In the flat-bed condition, owing to the generation of bed turbulence, the larger Reynolds stress is observed in the mid-depth layer than in the free-surface. Through the passing of the shedding vortices accompanied by the upward currents, large turbulence structure near the bottom layer is transported toward the free-surface. The turbulence is diffused during this vertical transport process, and thus, it becomes smaller nearer the free-surface. The Reynolds stress in the free-surface is obviously larger in the downward-slope bed condition than in the flat one. This may be because the distance between the flume bottom and the free-surface is very small in the upward-side of the embayment, and then, larger turbulence is generated and convected downstream.

4) Concentration distributions trapped in the embayment have significant relation with the formations of the PG and the SG in the flat-bottom and the downward-bottom conditions. In the PG zone where the mass exchange is strikingly conducted, no concentration injected at the initial stage is observed and the dye is concentrated in the SG zone. In the case of upward slope, a high concentration region with elliptical shape is organized from the upstream to midstream in the cavity. This elliptical zone is out of contact with three sidewalls within the cavity, and it exists in the center of the large-scale PG

5) We could obtain the linear relation between the mass transfer velocity and Reynolds stress in the mixing layer, irrespective of the bed slope conditions. This suggests that the Reynolds stress plays most significant roles on the mass transfer beneath the mainstream and the embayment.

\section{ACKNOWLEDGEMENT}

The present study was carried out under the financial support from the Research Project Grant-In-Aid for Scientific Research of Japanese Government (Grant-in-aid for young scientists No. 23760458, Principal Investigator M. Sanjou). The authors gratefully acknowledge this support. The authors would also like to thank S. Suzuki, K. Itai, M. Ikeuchi and K. Sakitani for their great help in the experiments and data analysis. 


\section{REFERENCES}

Akkerman, G.J., van Heereveld, M.A., van der Wal, M. and Stam, J.M.T.: Groyne optimization and river hydrodynamics, Proc. of Riverflow2004, Napoli, in CD-ROM, 2004.

Booij, R.: Laboratory experiments of shallow free surface flows, Shallow Flows Vol.2, Jirka \& Uijttewaal eds., pp.317-323, 2004.

Borg, A., Bolinger, J. and Fuchs, L.: Simultaneous velocity and concentration measurements in the near field of a turbulent low-pressure jet by digital particle image velocimetry-planar laser-induced fluorescence, Exp fluids Vol.31, pp.140-152, 2001.

Chong, M. S. and Perry, A. E. 1990. A general classification of three-dimensional flow fields, Phys. Fluids, A, Vol.2, pp.765-777.

Constantinescu, G., Sukhodolov, A. and McCoy, A.: Mass exchange in a shallow channel flow with a series of groynes: LES study and comparison with laboratory and field experiments, Environmental Fluid Mechanics, Vol.9, pp.587-615, 2009.

Cowen, E.A., Chng, K.A. and Liao, Q.: A single-camera coupled PTV-LIF technique, Experiments in Fluid, Vol.31, No.1, PP.63-73, 2001.

Duan, J.G., He, L., Fu, X. and Wang, Q.: Mean flow and turbulence around experimental spur dyke, Advances in Water Resources, Vol.32, pp.1717-1725, 2009.

Ercan, A. and Younis, B.A.: Prediction of bank erosion in a reach of the Sacramento river and its mitigation with groynes, Water Resour Manage, Vol.23(15), pp.3121-3147, 2009.

Ettema, R. and Muste, M.: Scale effects in flume experiments on flow around a spur dyke in flatbed channel, J. of Hydraulic Engineering, Vol.130(7), 635-646, 2004.

Gu, Z., Akahori, R. andykeda, S.: Study on the transport of suspended sediment in an open-channel flow with permeable spur dyke, Int. J. Sediment Research, Vol.26, pp.96-111, 2011.

Gulliver, J. S. \& Halverson, M.J.: Air-water gas transfer in open channels, Water Resources Research, Vol.25(8), pp.1783-1793, 1989.

Kadota, A. \& Suzuki, K.: Local scour and development of sand wave around a permeable groyne of stone gabion, Environmental Hydraulics, Christodoulou \& Stamou (eds.), 807-812, 2010.

Mioduszeski, T. and Maeno, S.: Influence of the structure permeability on scouring and flow around a spur dyke during a surge pass, Journal of Applied Mechanics, JSCE, Vol.9, pp.853-864, 2006.

Nezu, I. and Nakagawa, H.: Turbulence in Open - Channel Flows, IAHR Monograph, Balkema, 1993.

Nezu, I. and Sanjou, M.: PIV and PTV measurements in hydro-sciences with focus on turbulent open-channel flows, Journal of hydro-environment research, Vol.5, pp.215-230, 2011.

Sukhodolov, A., Engelhardt, Kruger, A. and Bungartz, H: Case study: Turbulent flow and sediment distributions in a groyne field, Journal of Hydraulic Engineering, Vol.130(1), pp.1-9, 2004.

Tominaga, A. \& Sakaki, T.: Evaluation of bed shear stress from velocity measurements in 
gravel-bed river with local non-uniformity, Riverflow 2010, Dittrich, Koll, Aberle \& Geisenhainer (eds), 187-194, 2010.

Uijttewaal, W.S.J., Lehmann, D. and van Mazijk, A.: Exchange processes between a river and its groyne fields: Model experiments, Journal of Hydraulic Engineering, Vol.127(11), pp.928-936, 2001.

Uijttewaal, W.S.J.: Effects of groyne layout on the flow in groyne fields: Laboratory experiments, Journal of Hydraulic Engineering, Vol.131(9), pp.782-791, 2005.

Valentine, E.M. and Wood, I.R.: Longitudinal dispersion with dead zones, Journal of Hydr. Div. Vol.103(9), pp.975-990, 1977.

Weitbrecht, V, Socolofsky, S.A. and Jirka, G.: Experiments on mass exchange between groin fields and main stream in rivers, Journal of Hydraulic Engineering, Vol.134(2), pp.173-183, 2008.

Westerweel, J. Efficient detection of spurious vectors in particle image velocimetry data, Experiments in Fluid, Vol.16, pp.236-247, 1994.

Yossef, M.F.M. and de Vriend, H.: Flow details near river groynes: Experimental investigation, Journal of Hydraulic Engineering, Vol.137(5), pp.504-516, 2011.

Zhen, Q., Zhang, W. and Jin, X.: Analysis for reliability of groyne safety during random erosion process, Proc. of 2009 World congress on computer science and information engineering, Los Angeles, pp.139-143, 2009. 


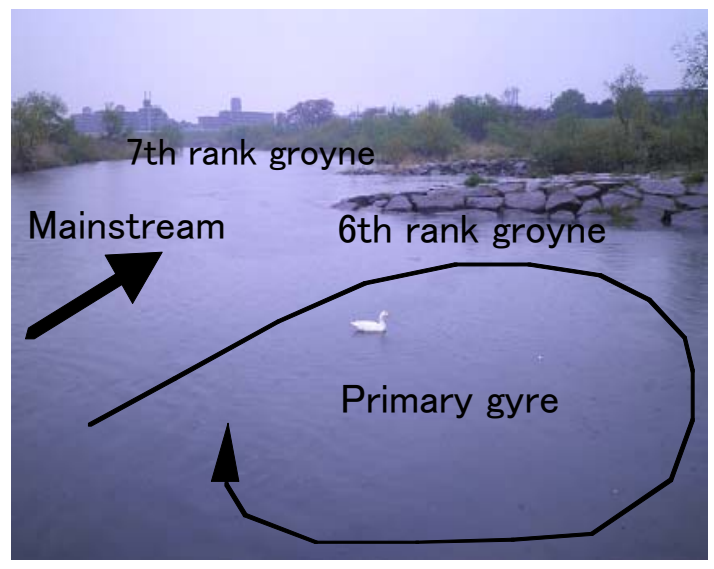

Photo 1 Example of a consecutive groyne field, Katsura River in Kyoto, Japan 


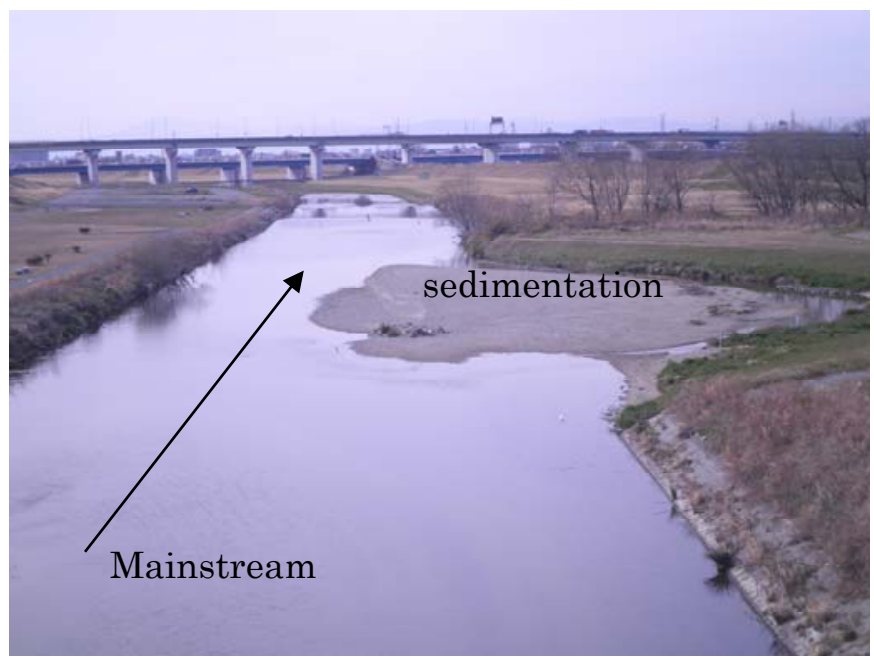

Photo2 The sedimentation observed in the mainstream/embayment boundary in a natural river, the Yahagi River in Nagoya, Japan 
(a)
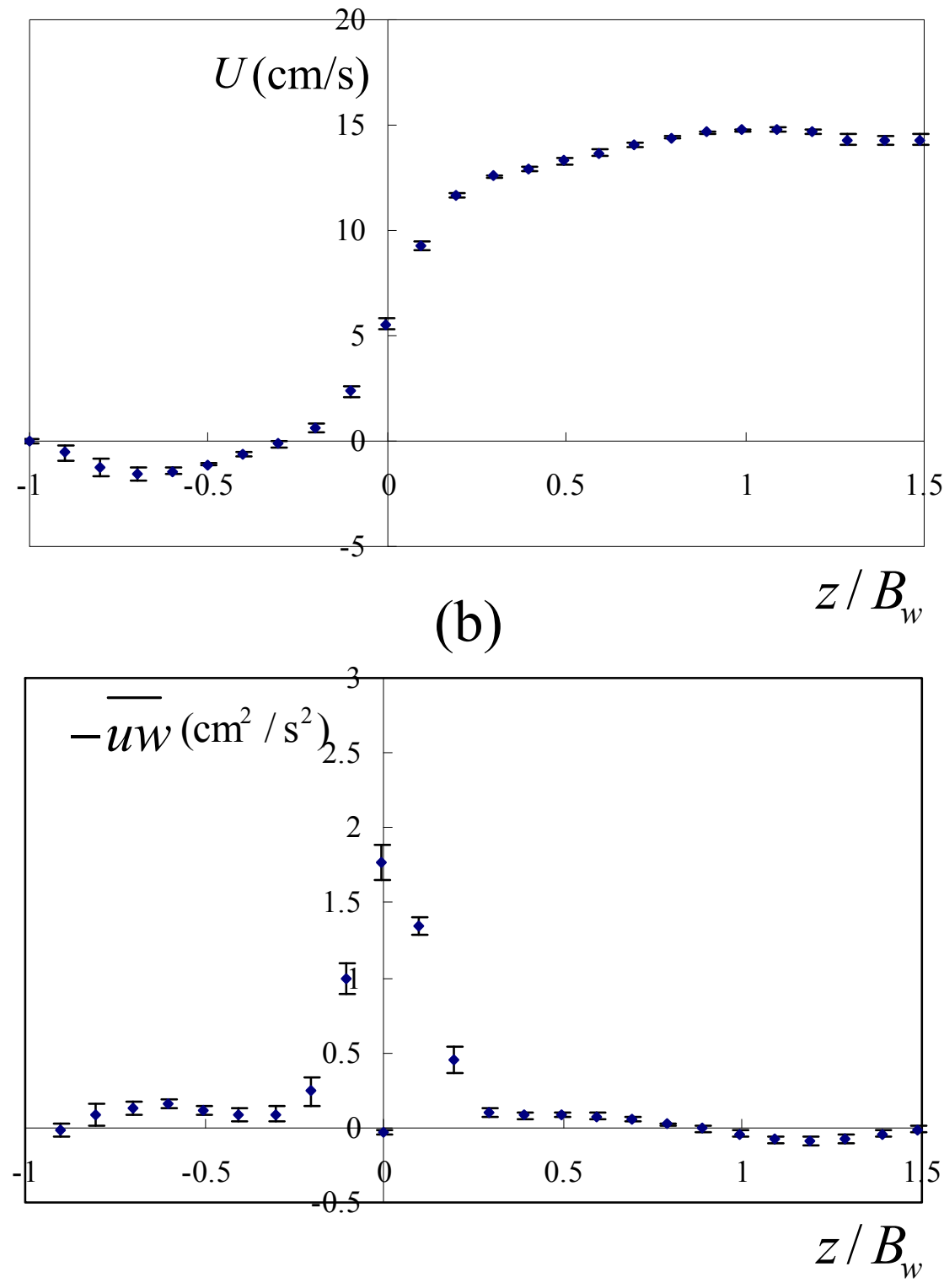

Fig.1 The examination of repeatability for the present PIV measurements (ensemble mean value and standard deviation profiles for a: time-averaged streamwise velocity, b: horizontal Reynolds stress) 
(a)

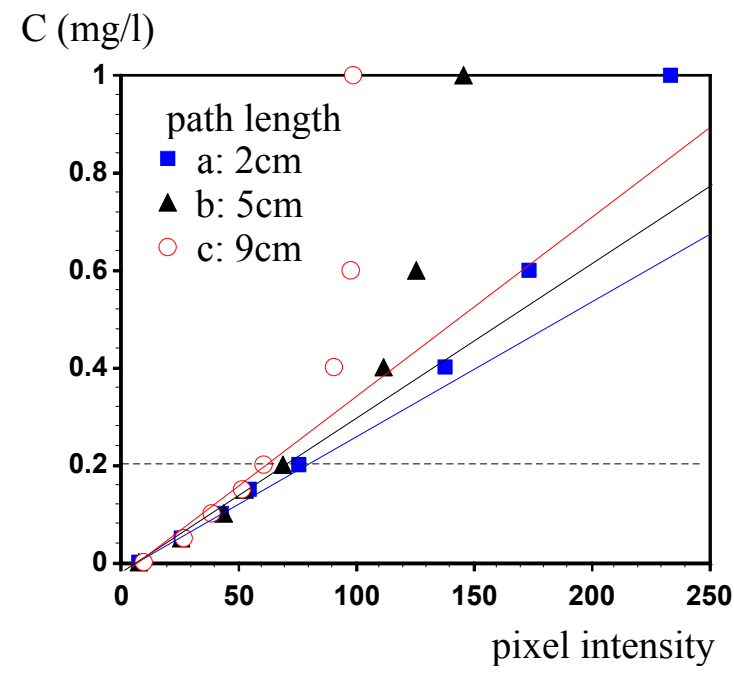

(b)

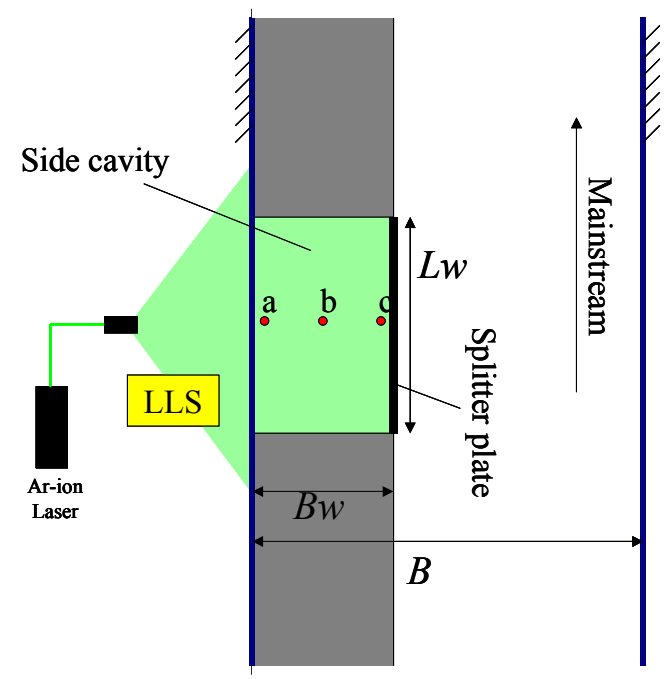

(c)

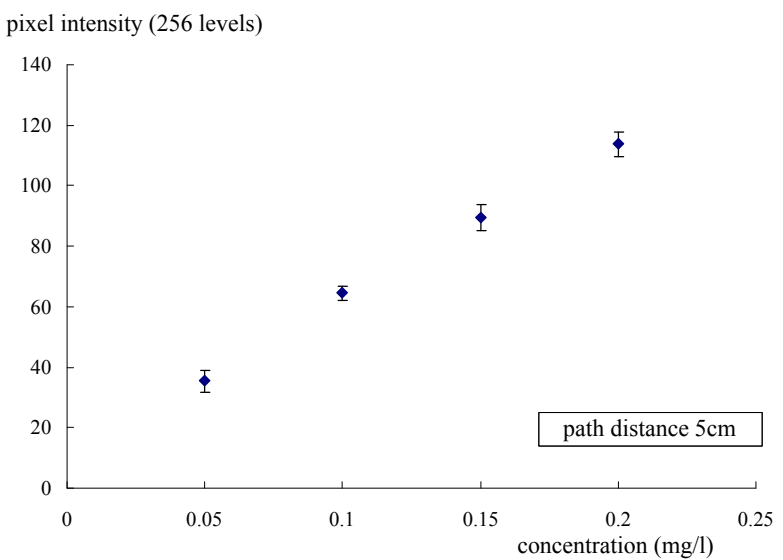

Fig.2 The calibration results for the LIF measurement (a: curves for pixel intensity vs. dye concentration, $\mathrm{b}$ : the test positions, $\mathrm{c}$ : profile of standard deviation for $5 \mathrm{~cm}$ path distance) 
(a) Flat bed condition $S=0$

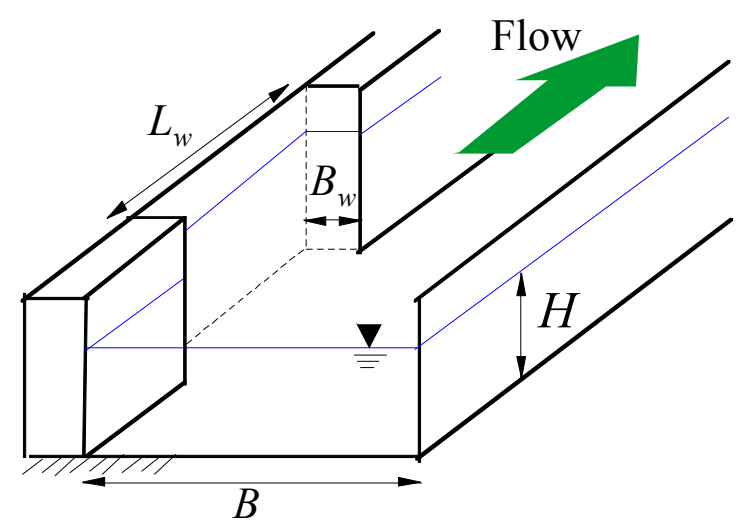

(b) Downward slope condition

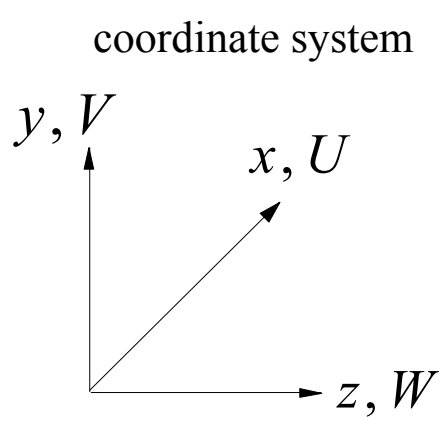

(c) Upward slope condition

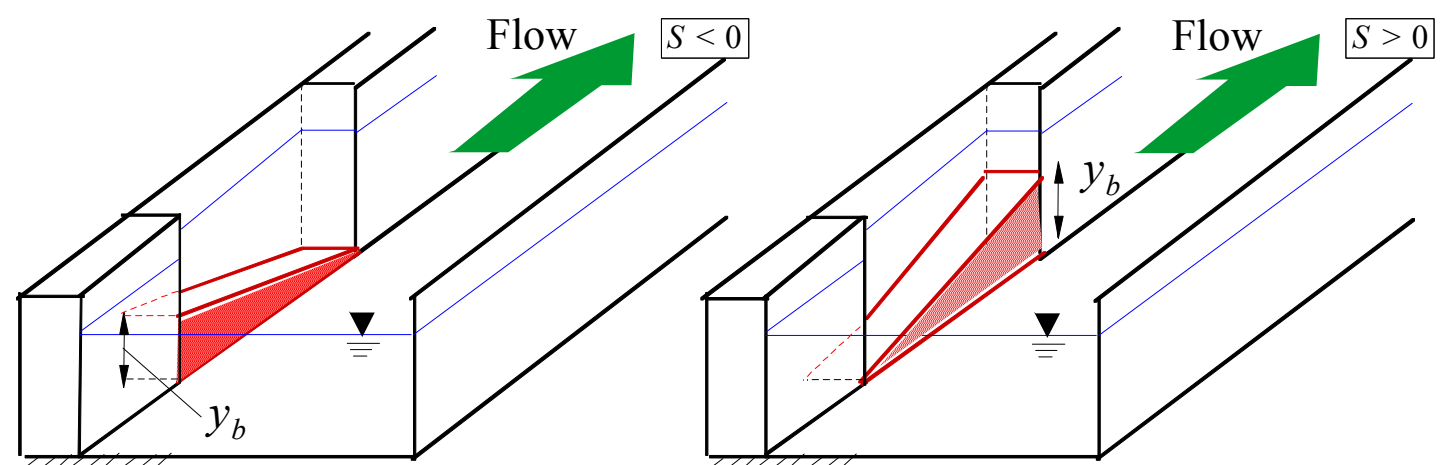

Fig.3Variation in the bed configuration within the embayment zone, as well as the coordinate system 

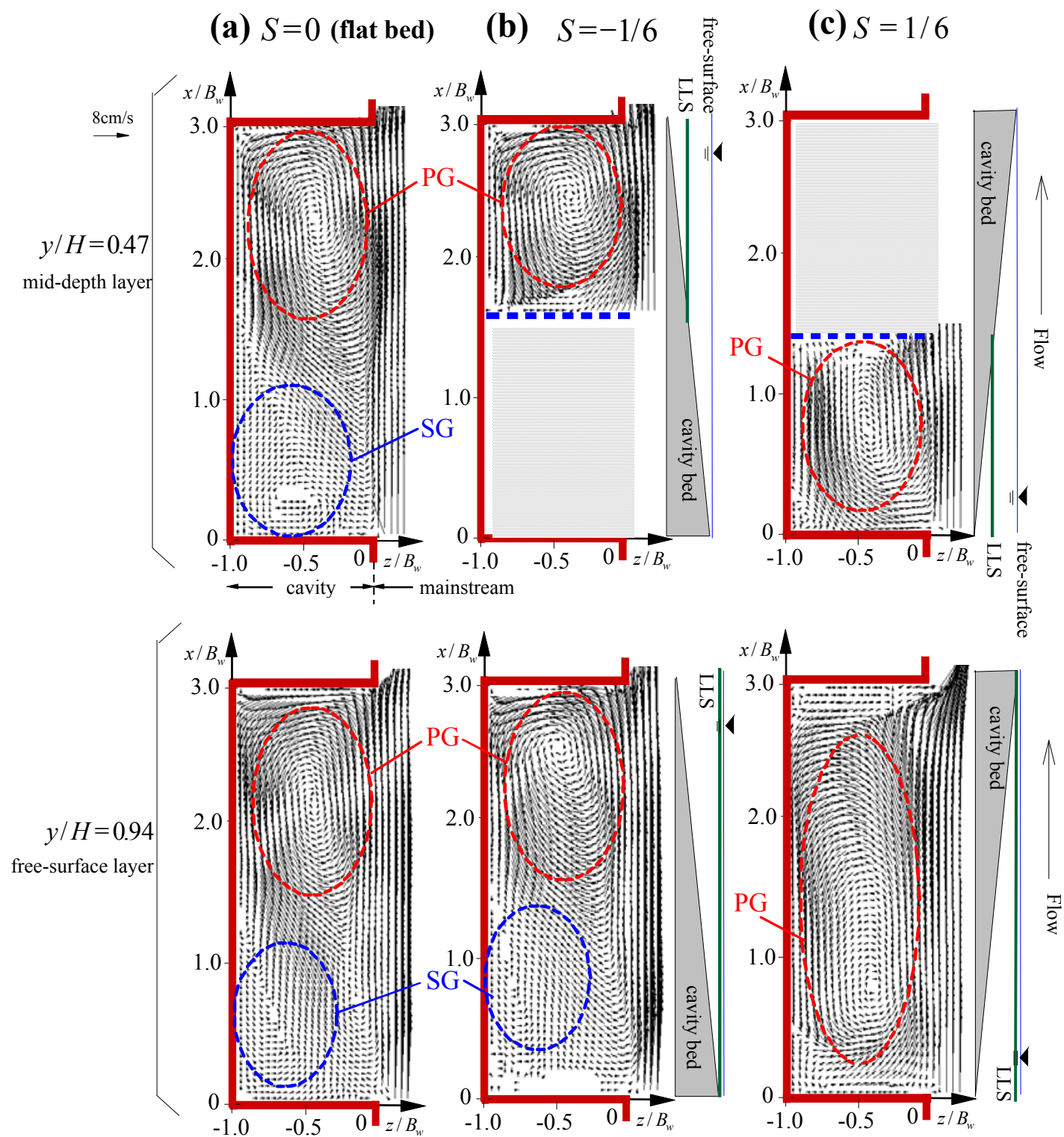

Fig.4 A comparison of time-averaged horizontal velocity components among different bed configurations 


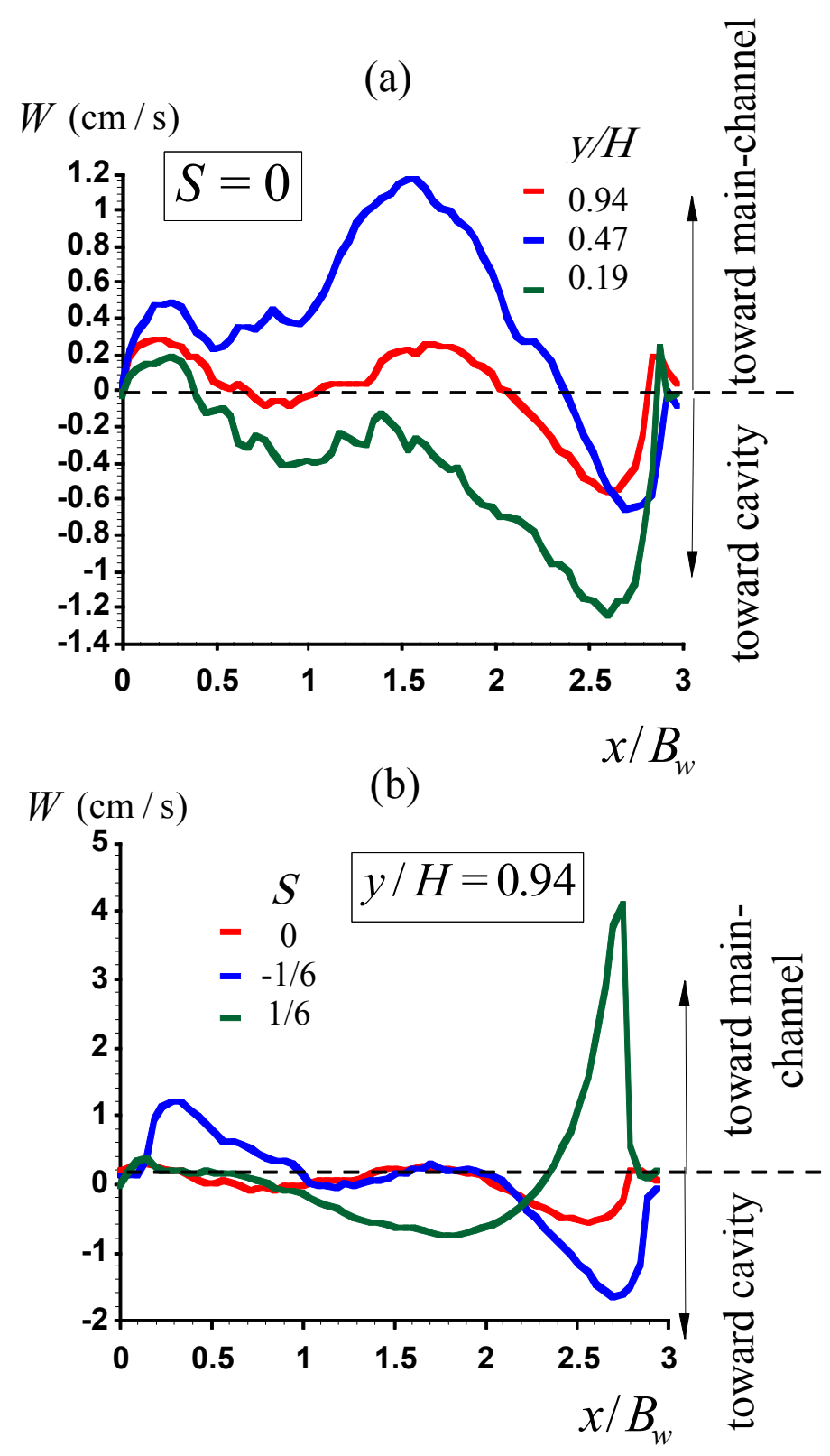

Fig.5 The streamwise variation of the time-averaged spanwise velocity along the main-channel/embayment boundary. a) A comparison for different elevations in the flat-bottom case, b) a comparison for different bed configurations near the free-surface 


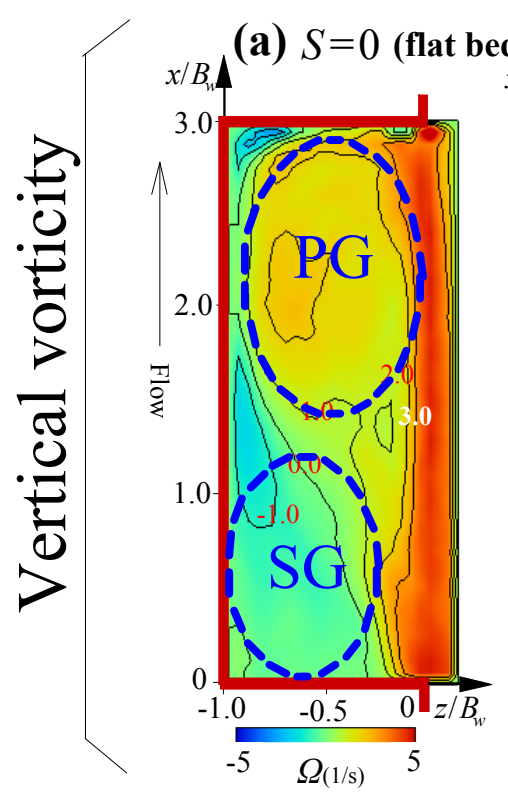

(b) $S=-1 / 6$

(c) $S=1 / 6$
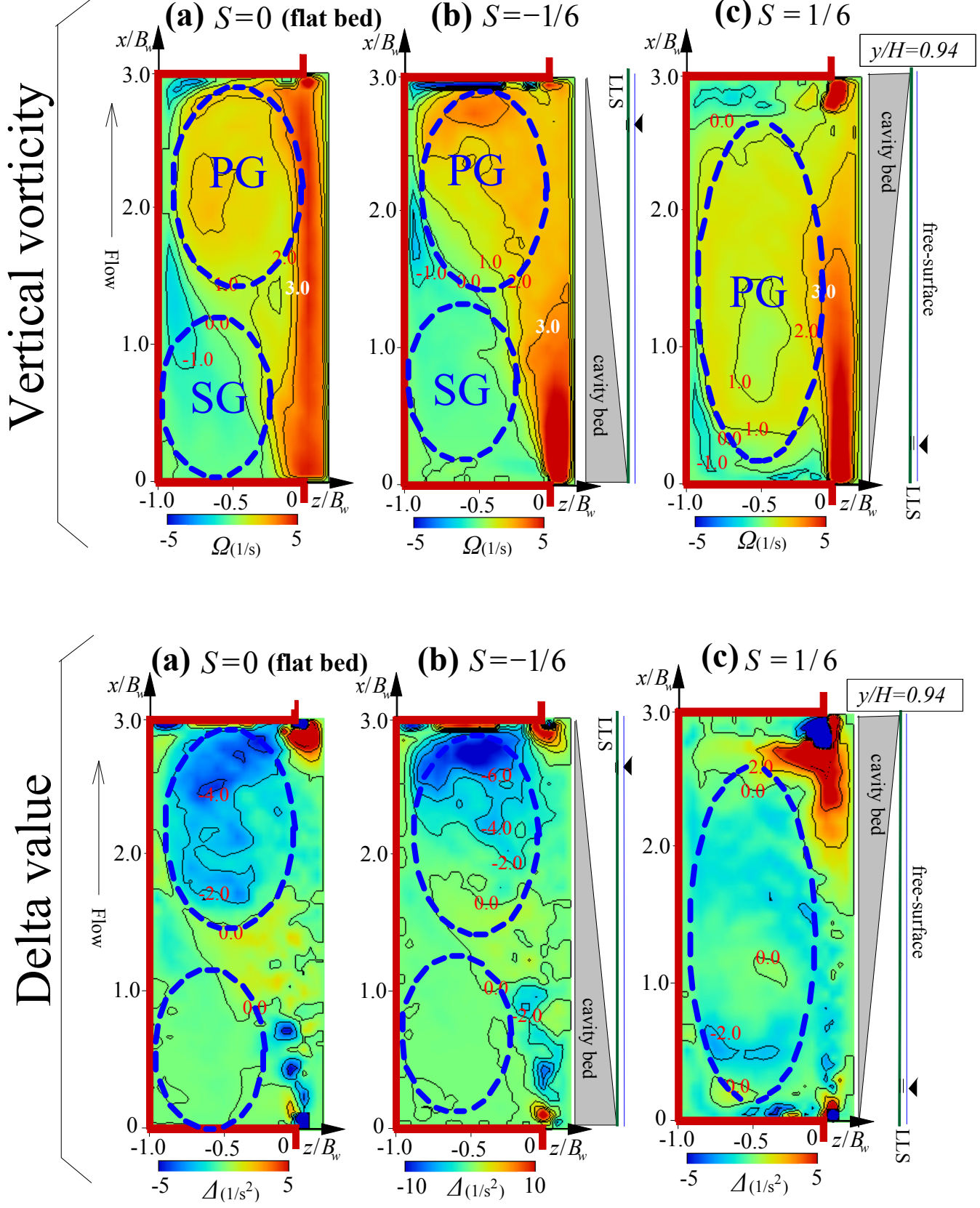

Fig.6 The horizontal distributions of vertical vorticity and delta values within the time-averaged field 

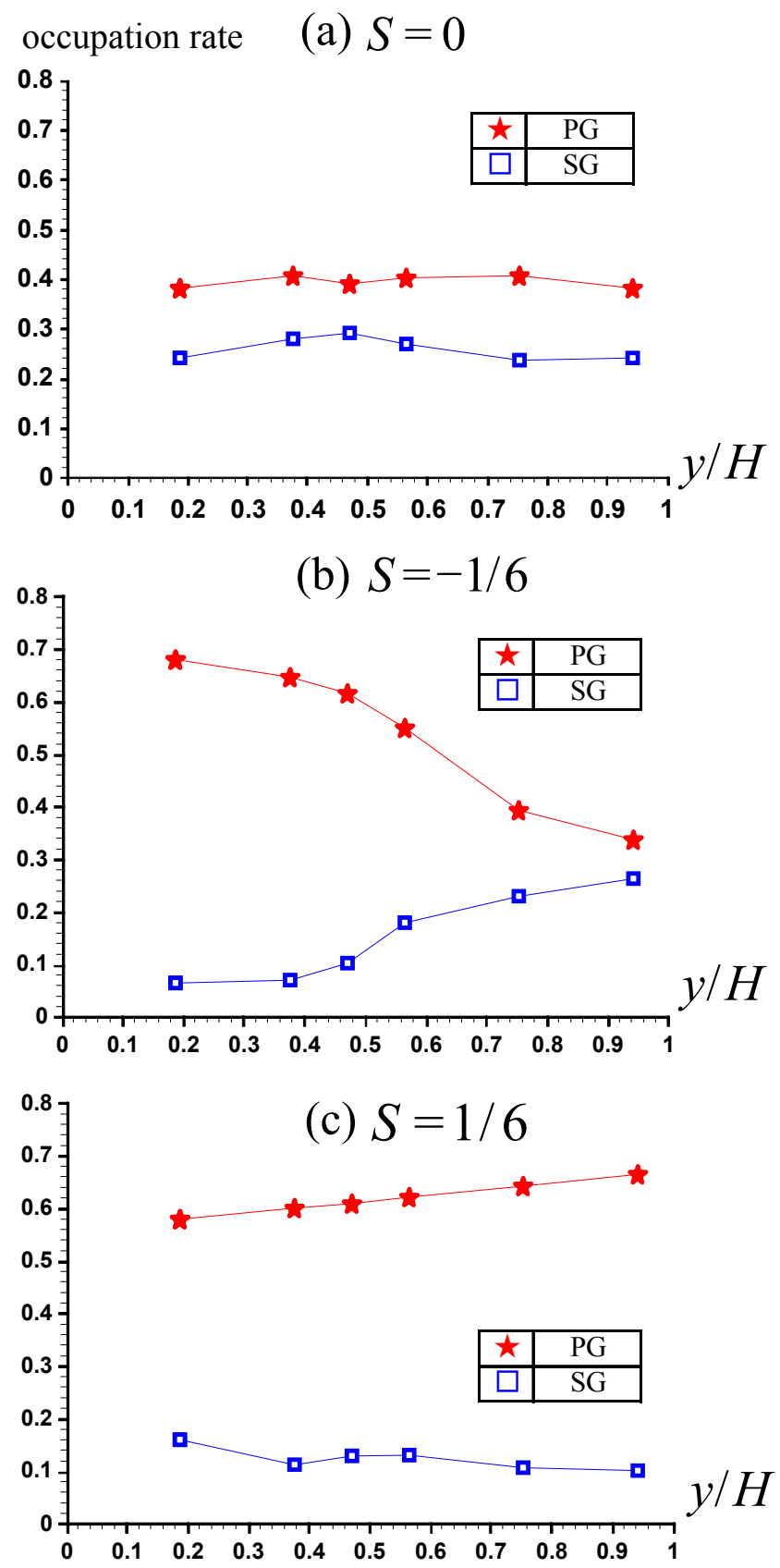

Fig. 7 The vertical profiles of the area occupation rate for primary and secondary gyres 

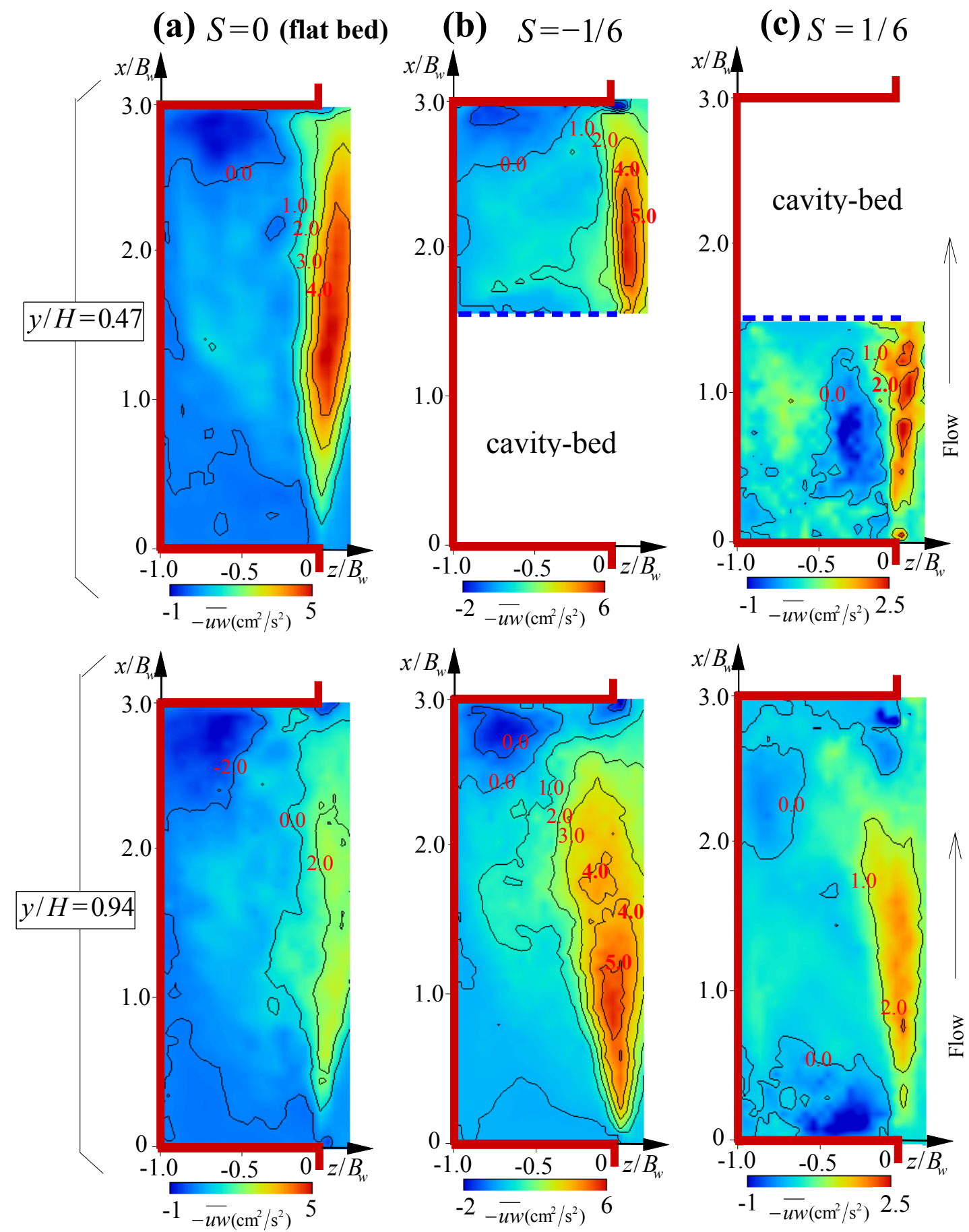

Fig.8 The horizontal distributions of the Reynolds' stress; a comparison amongst the different bed configurations 


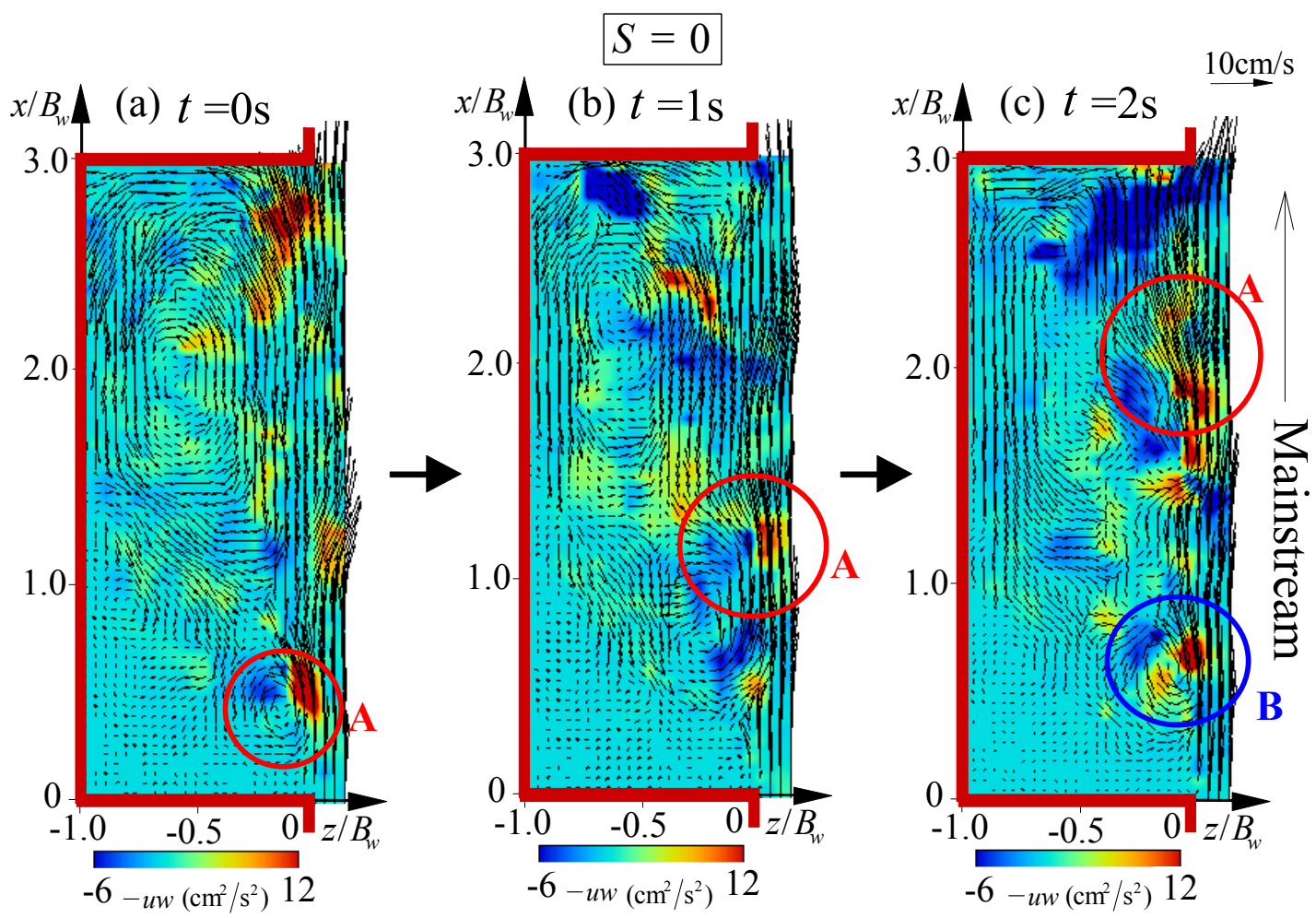

Fig.9(a) The time-series of the distributions of the instantaneous horizontal velocity vectors; contours indicate an instantaneous Reynolds stress (flat bed condition, $\mathrm{S}=0$ at $\mathrm{y} / \mathrm{H}=0.94)$ 


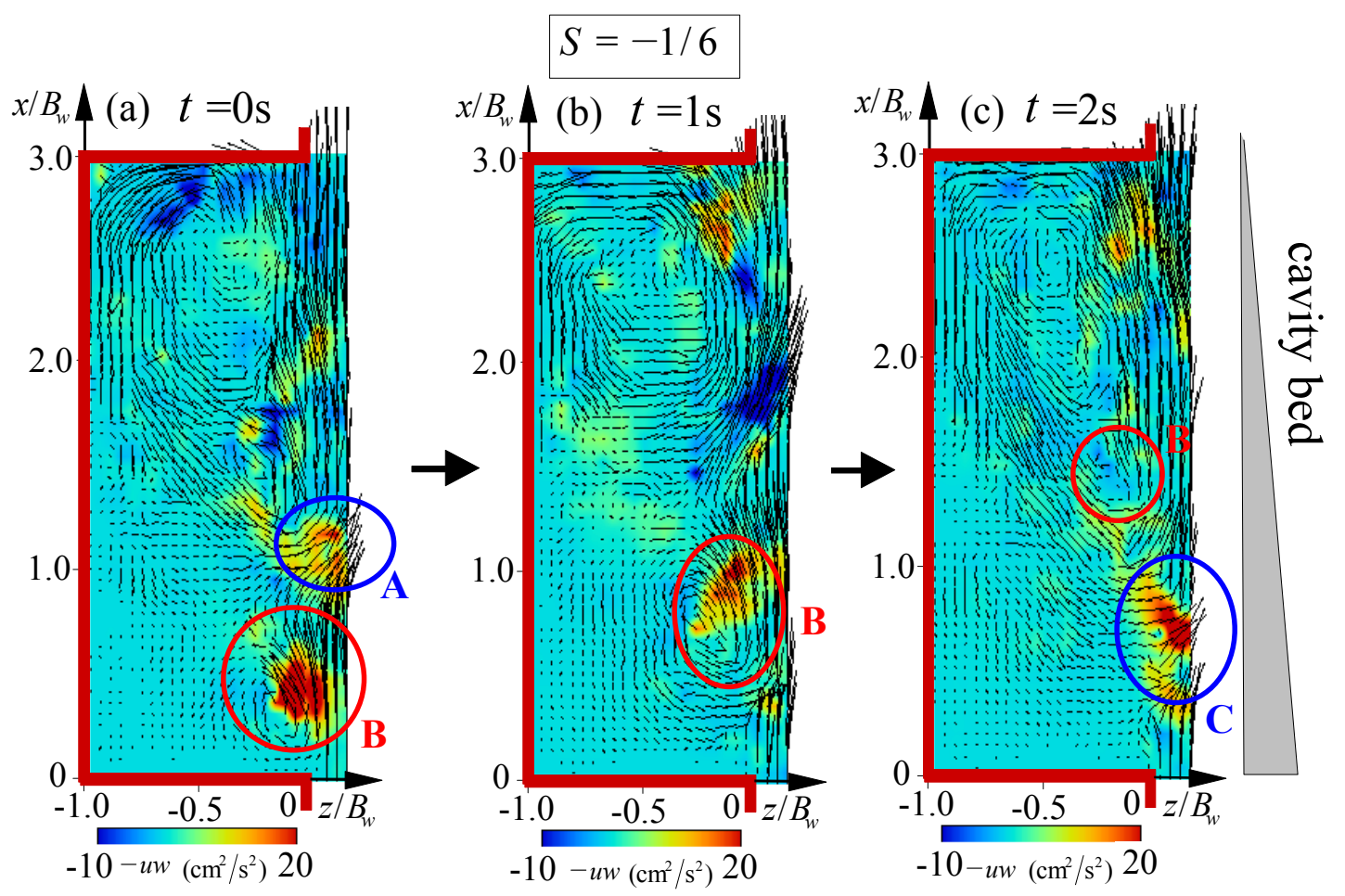

Fig.9(b) The time-series of distributions of instantaneous horizontal velocity vectors ; contour means instantaneous Reynolds stress (downward-slope bed condition, $S=-1 / 6$ at $y / H=0.94$ ) 


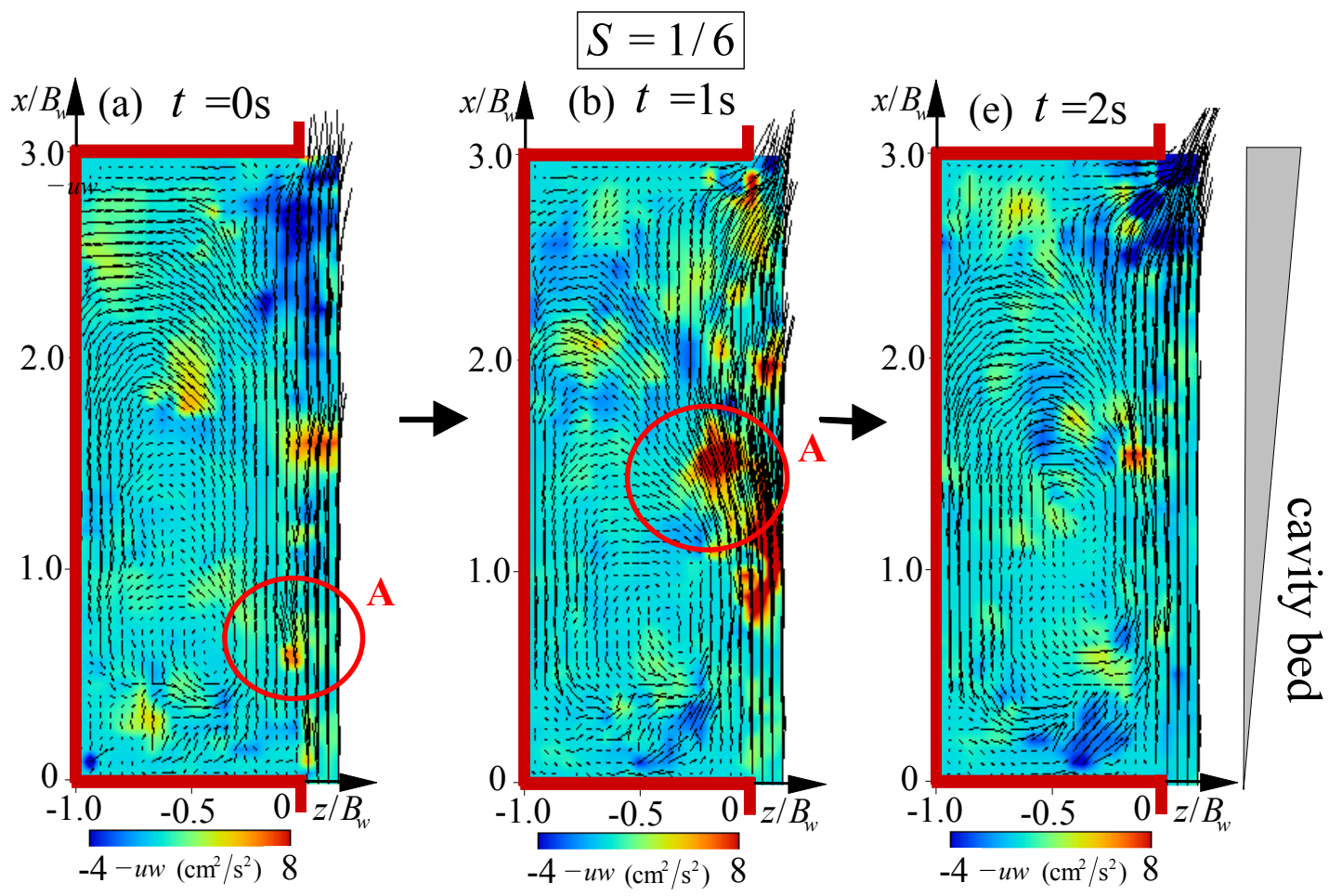

Fig.9(c) The time-series of distributions of instantaneous horizontal velocity vectors; contour means instantaneous Reynolds stress (upward-slope bed condition, $S=1 / 6$ at $y / H=0.94$ ) 


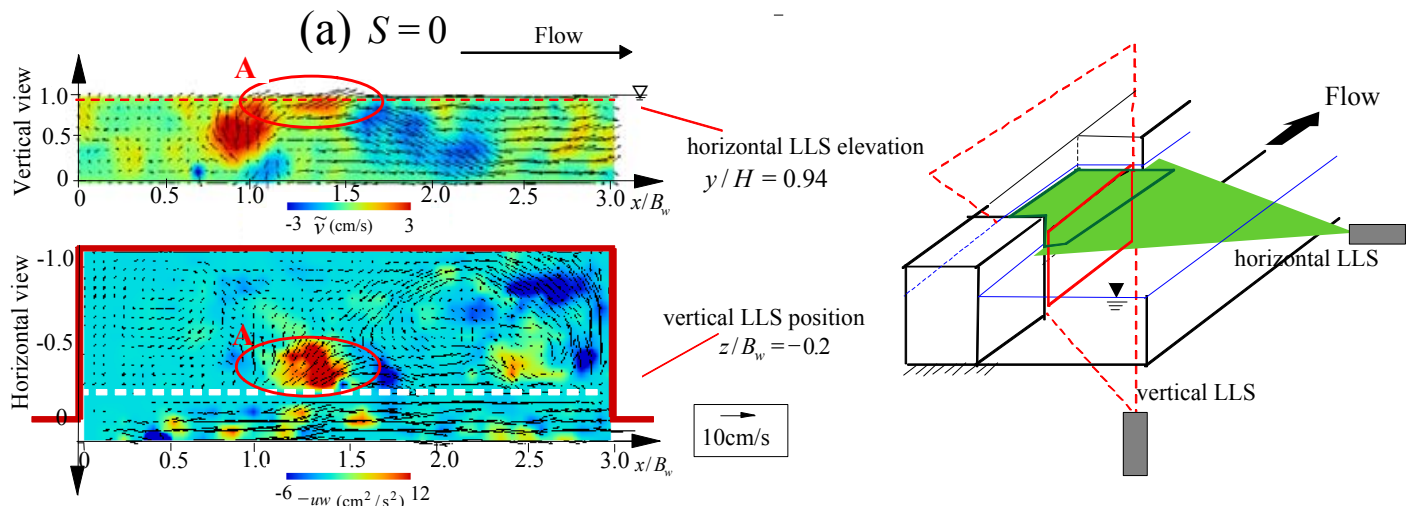

(b) $S=-1 / 6$

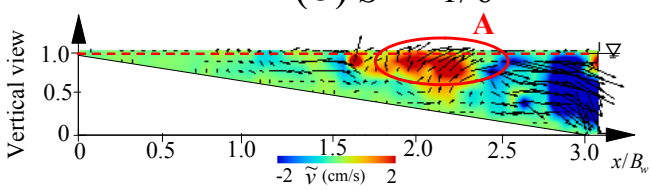

(c) $S=1 / 6$
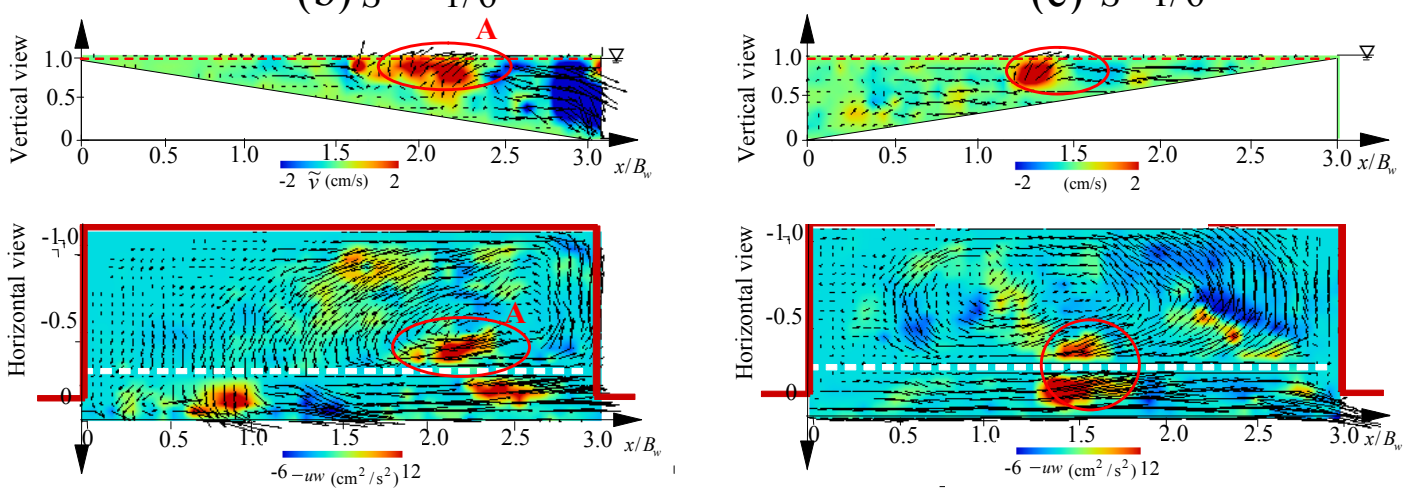

Fig.10 Examples of instantaneous velocity vectors in the vertical and horizontal planes; contours indicate the instantaneous vertical velocity component and Reynolds stress, respectively 
(a) $\tau=0 \mathrm{~s}$
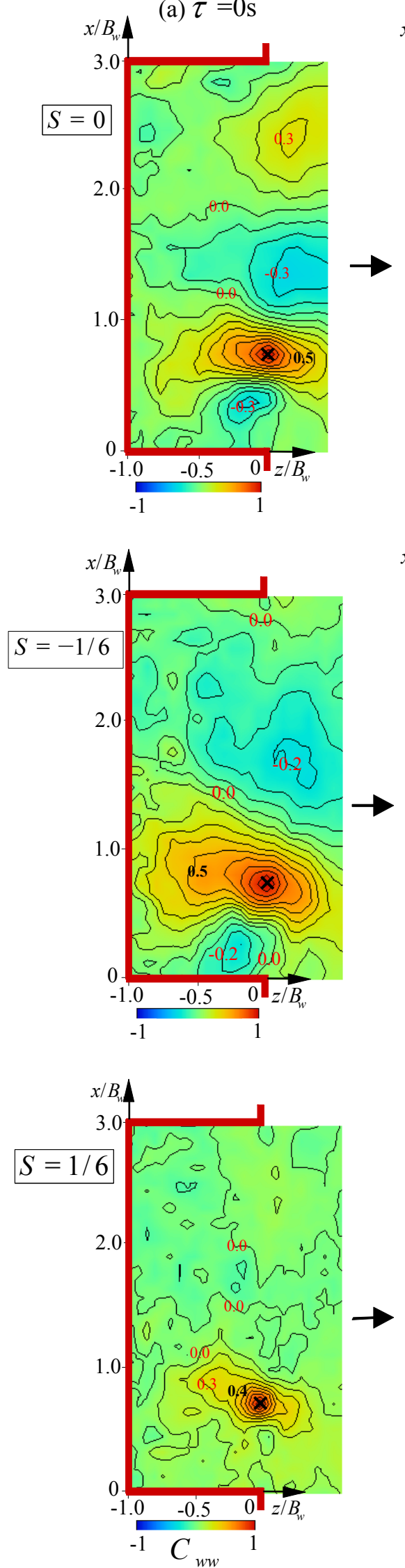

(b) $\tau=1.5 \mathrm{~s}$
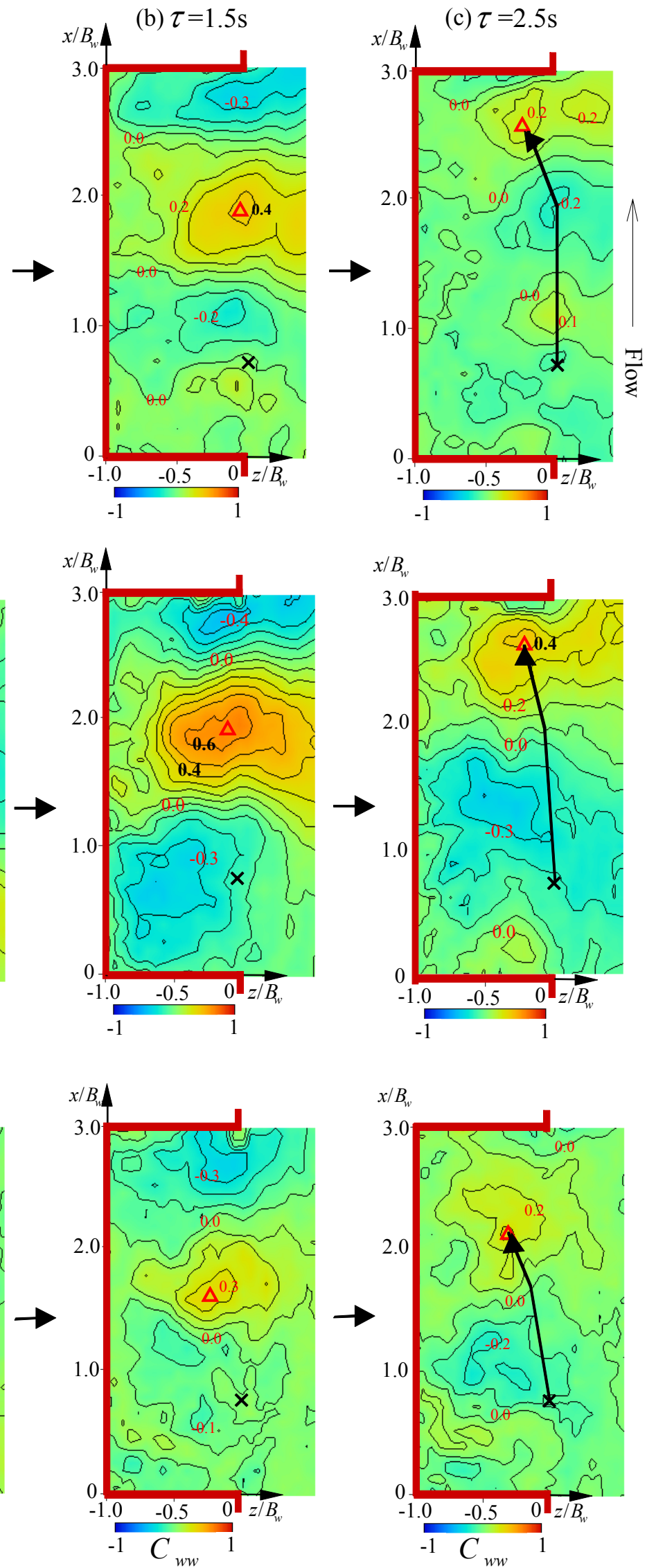

Fig.11 The space-time variations of the correlation function near the free-surface, $y / H=0.94$ 
(a) $\tau=0.0(\mathrm{~s})$

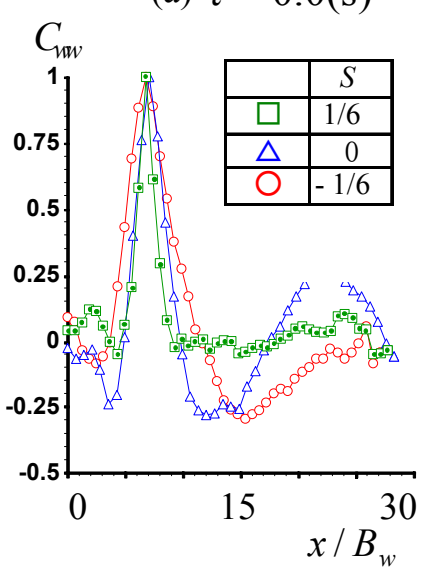

(b) $\tau=0.2(\mathrm{~s})$

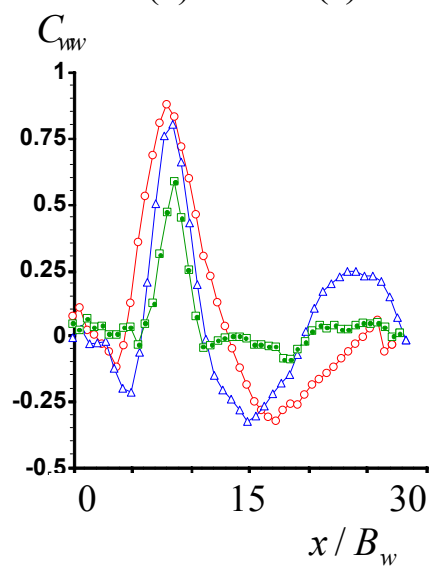

(c) $\tau=0.4(\mathrm{~s})$

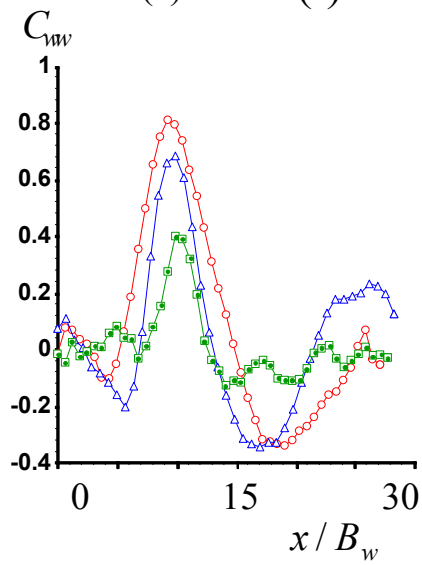

Fig.12 Longitudinal variations of the space-time correlation function along the junction 


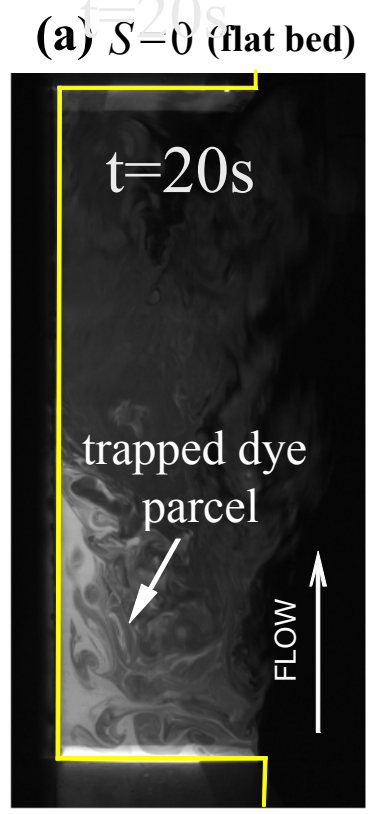

(b) $S=-1 / 6$
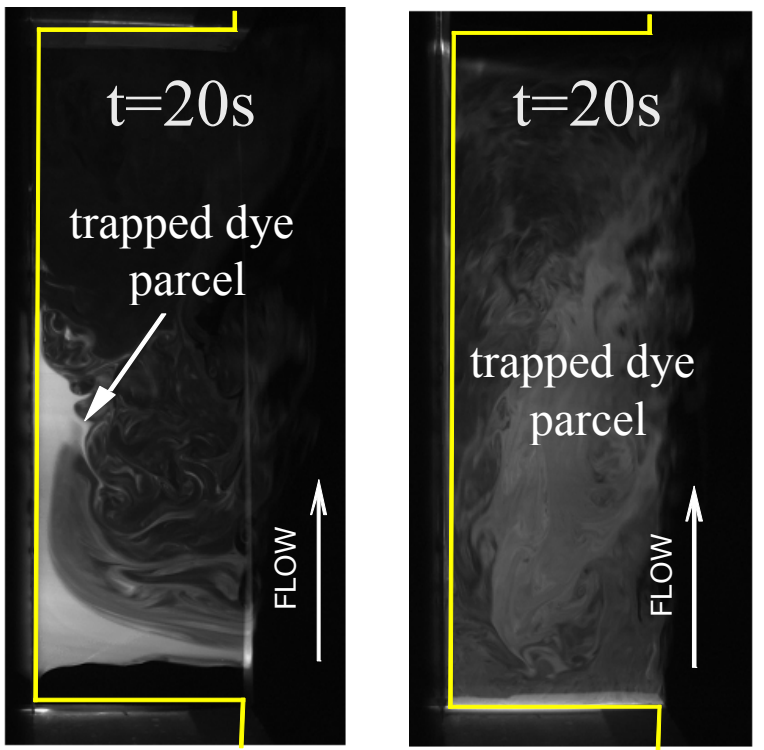

Fig.13 A comparison of the dye concentration trapped in the embayment 


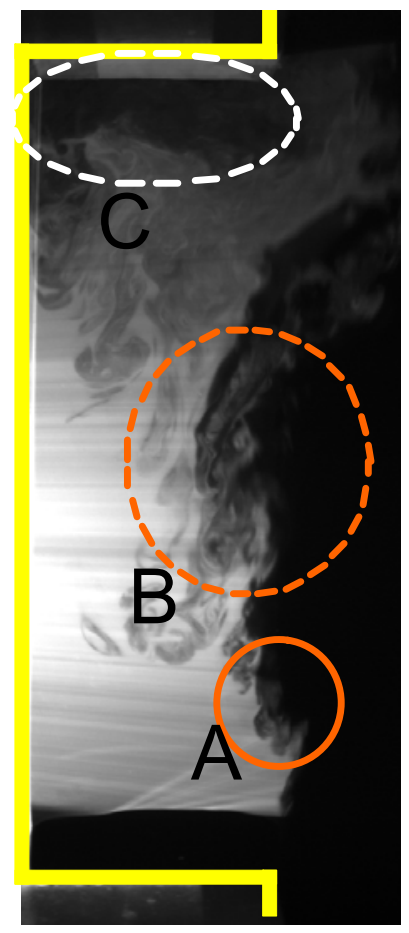

$\mathrm{t}=4.0(\mathrm{~s})$

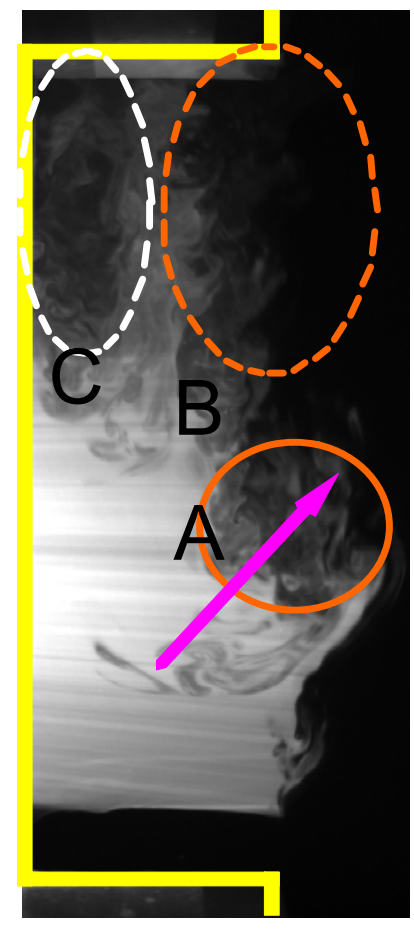

$\mathbf{t}=\mathbf{5 . 0}(\mathbf{s})$
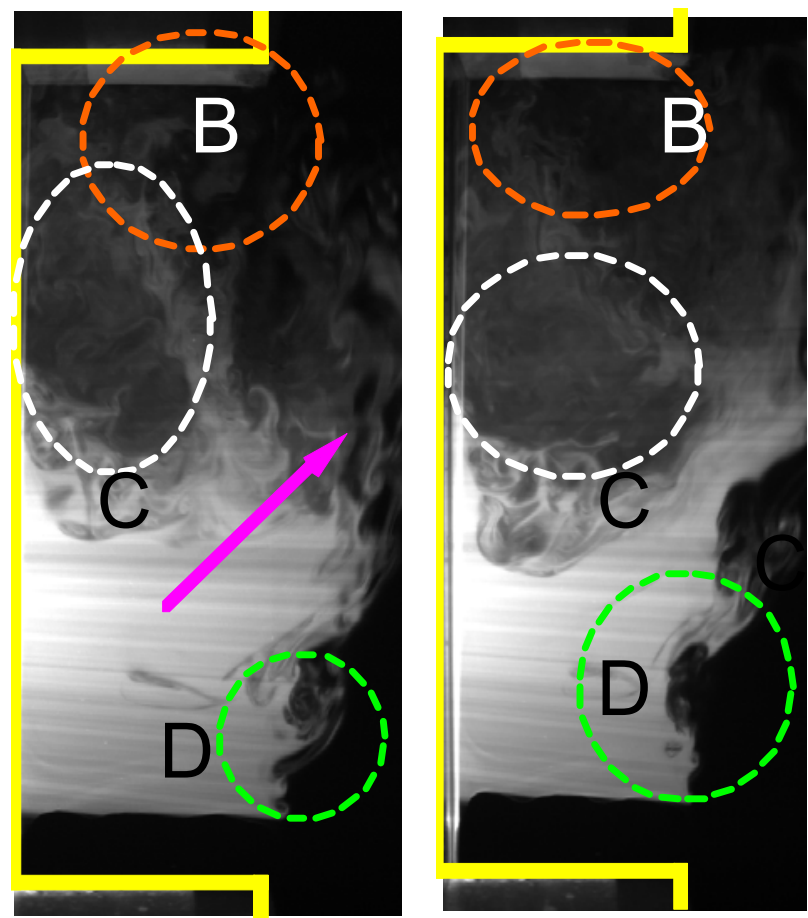

$\mathbf{t}=\mathbf{6 . 0}(\mathbf{s})$

$\mathrm{t}=7.0(\mathrm{~s})$

Fig.14 An example of the time-series of the dye concentration transport for the case of $S=-1 / 6$ 
(a)

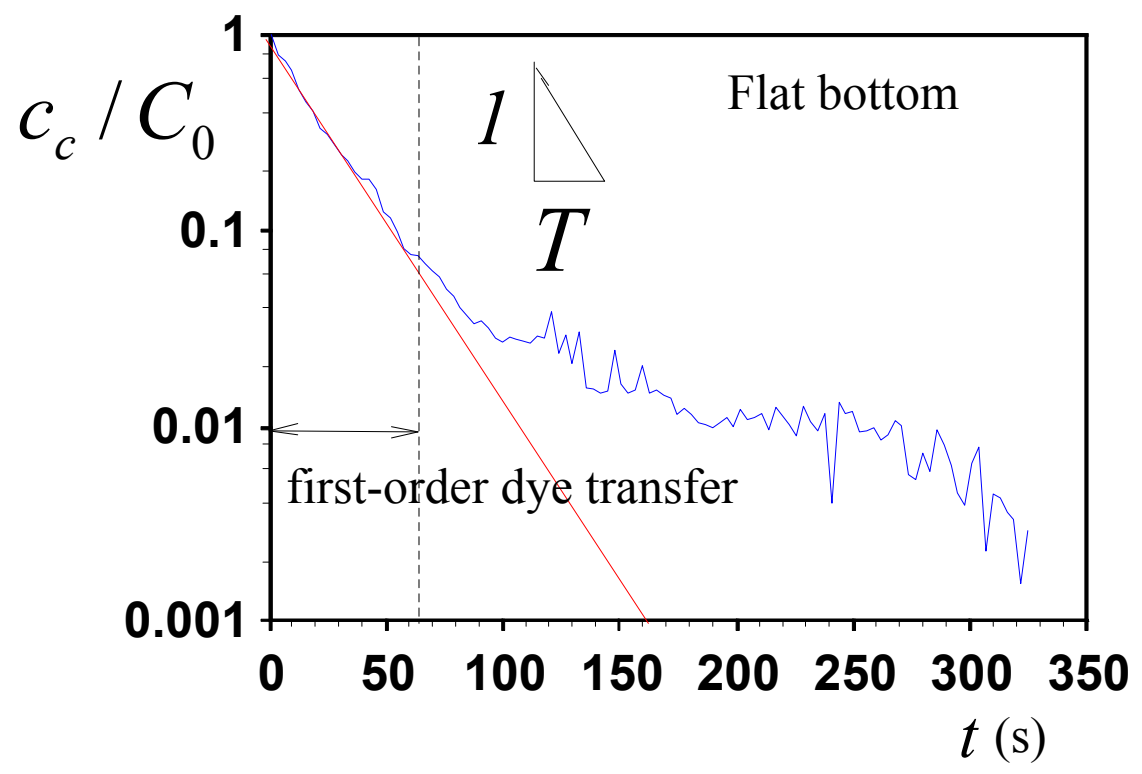

(b)

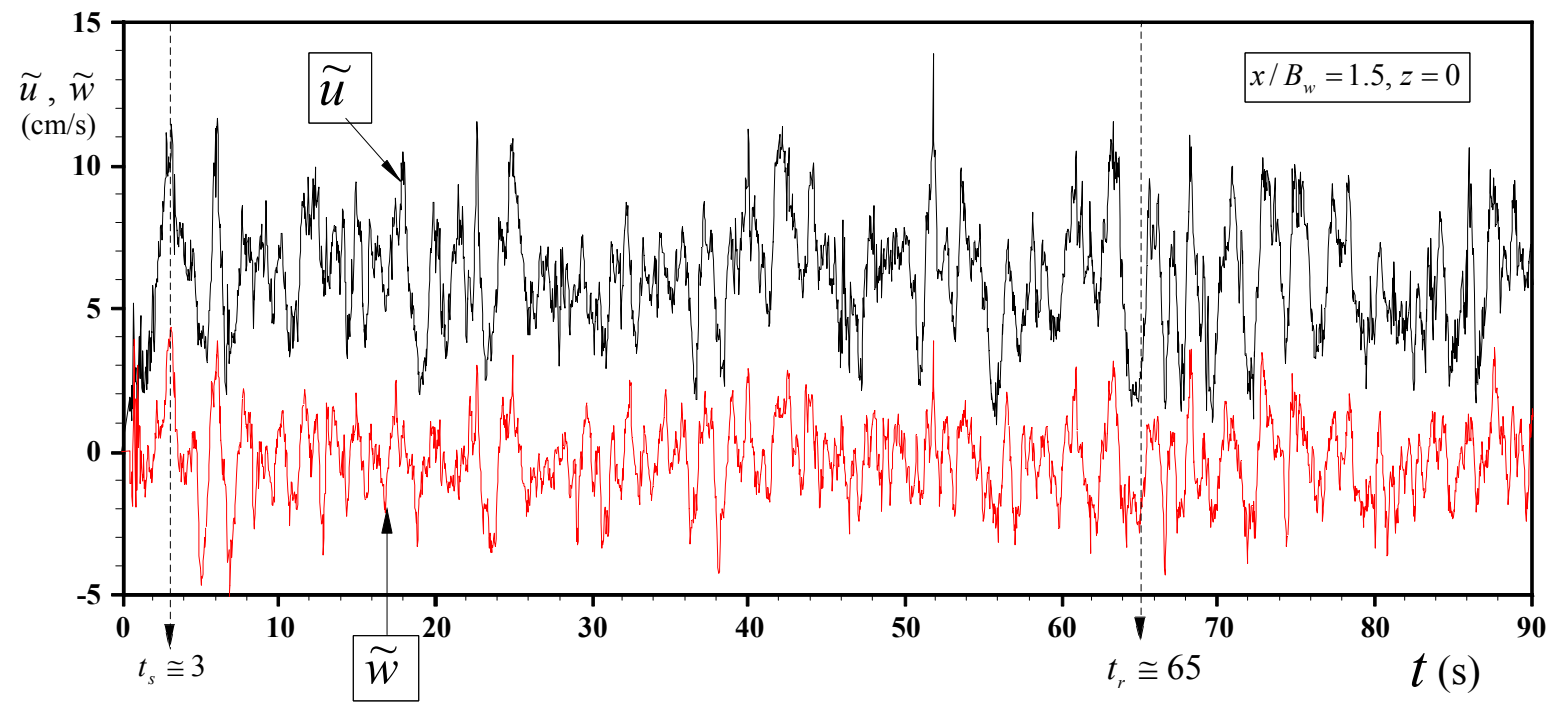

Fig. 15 The time decay of dye concentration and comparison of time-scales within the flat-bed embayment, $S=0$, for a: time-series of dye concentration, b: time-series of instantaneous velocity components with the spin-up and residence time-scales 


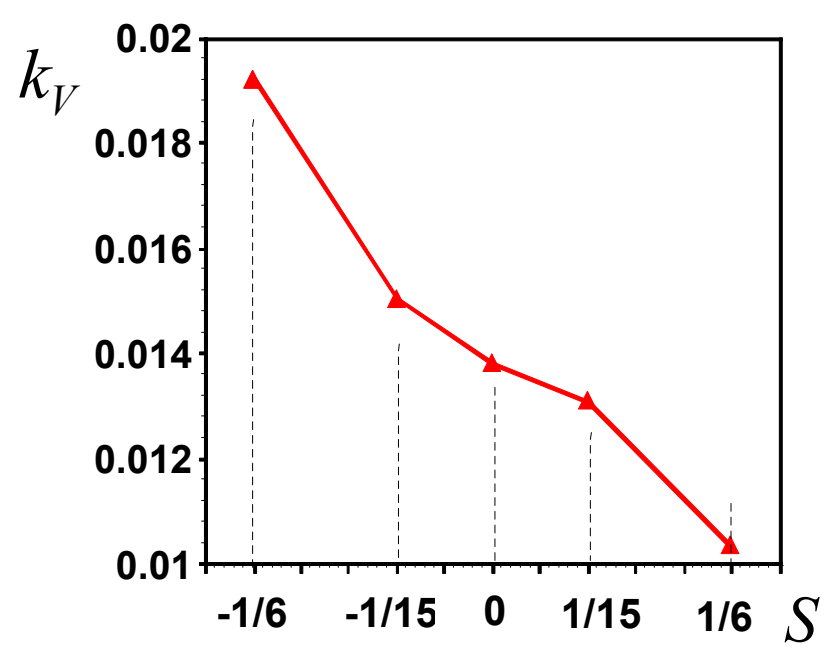

Fig.16 The relationship of the volume-averaged mass transfer velocity and the bed configuration 


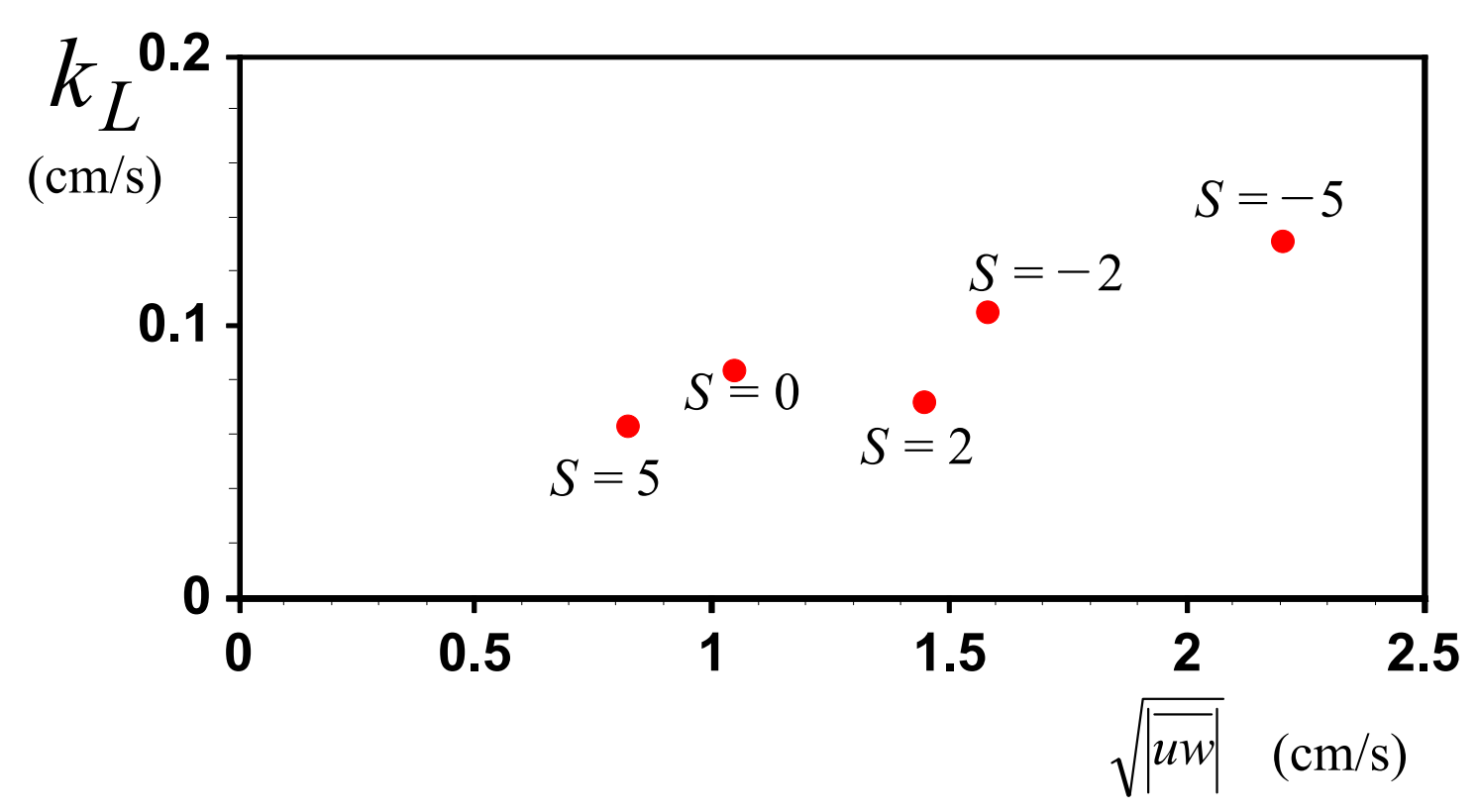

Fig.17 Relation between square root of Reynolds stress and transfer velocity 


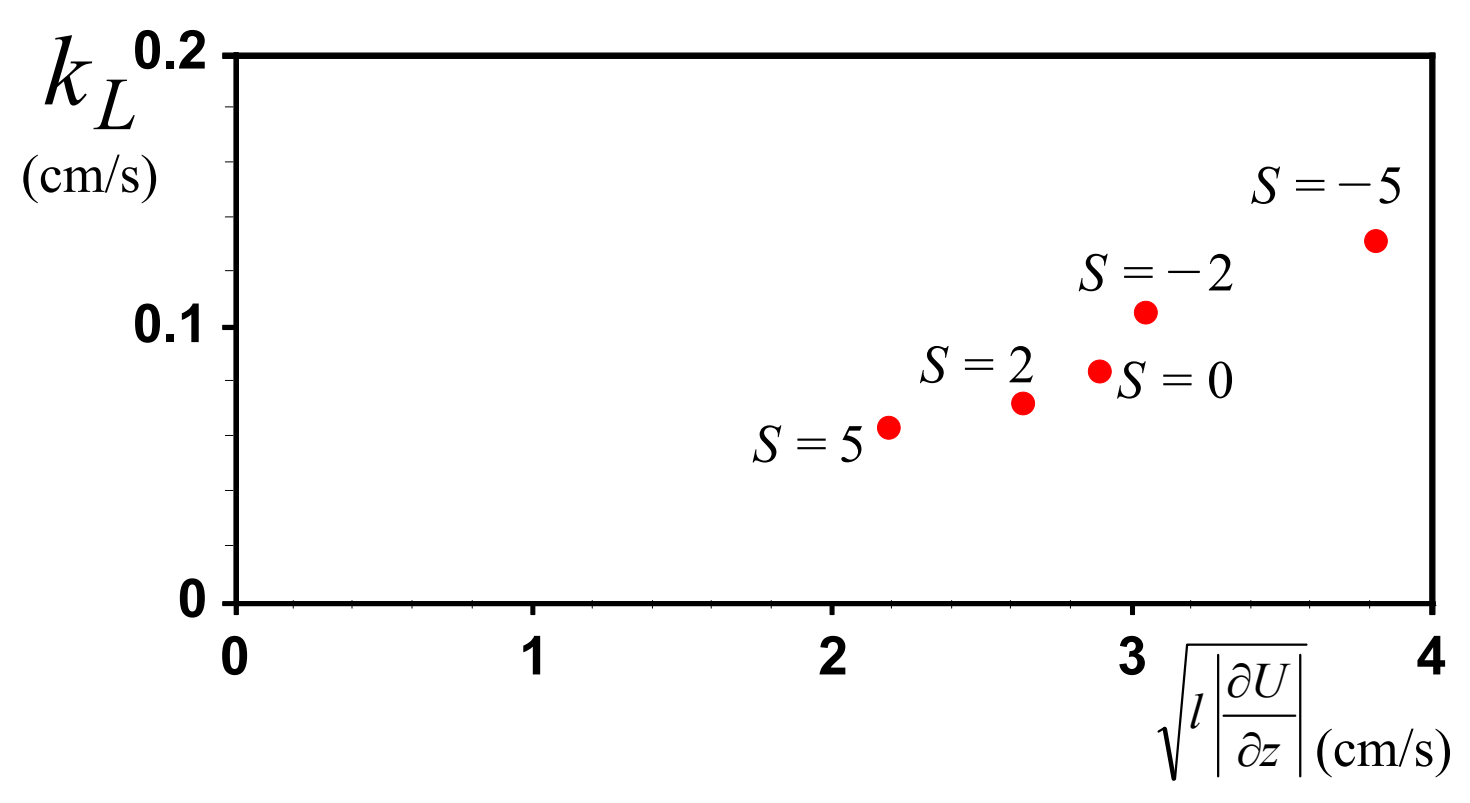

Fig.18 Relation between velocity shear and transfer velocity 
(a) Downward-slope bottom condition $(\mathrm{S}<0)$

(b) Upward-slope bottom condition ( $\mathrm{S}>0)$
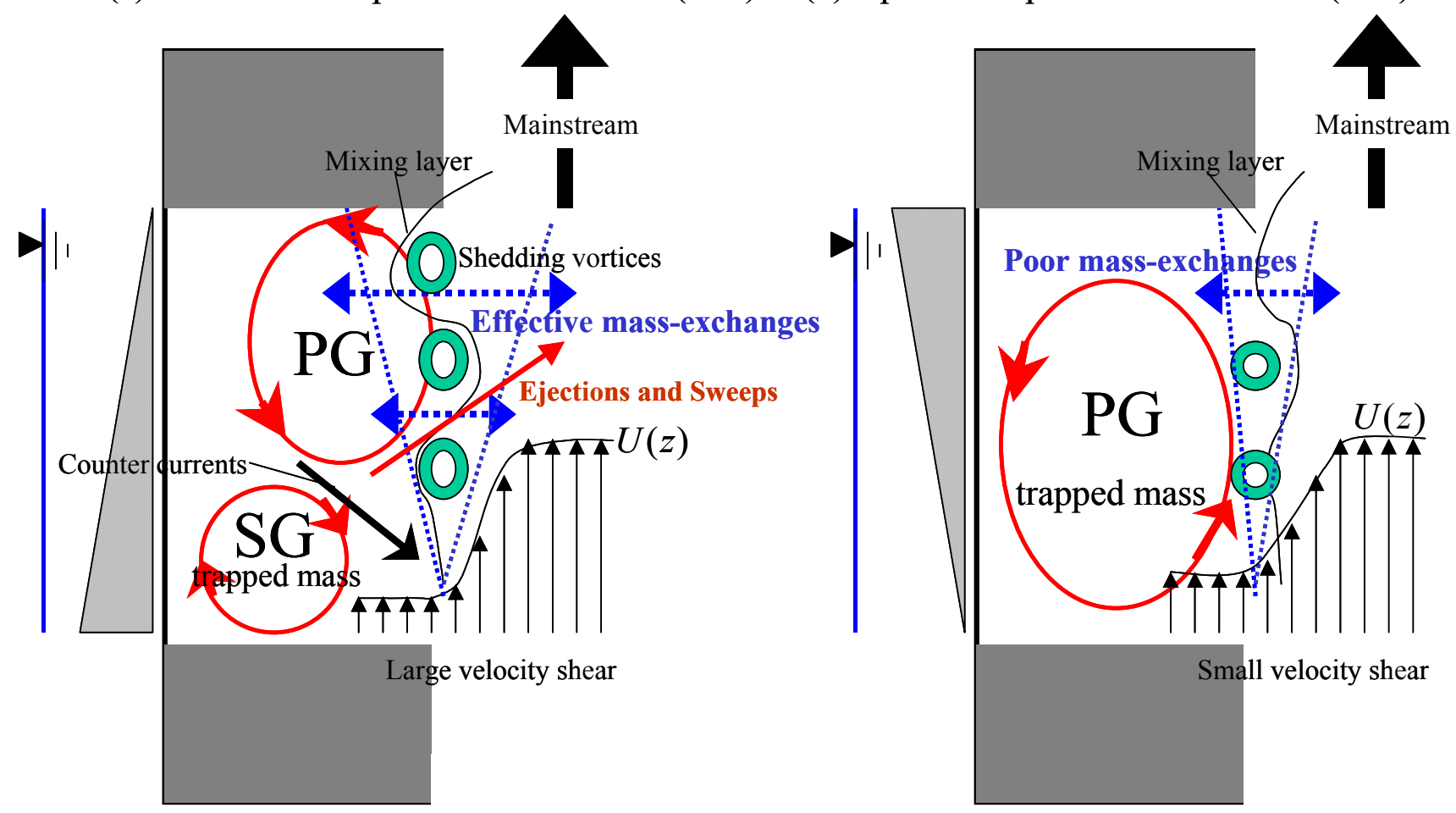

Fig.19 The phenomenological flow model for the dependencies of turbulence structure and mass transfer on the bed slope directions 
Table1 The hydraulic condition

\begin{tabular}{|c|c|c|c|c|c|c|c|}
\hline$U_{m}(\mathrm{~cm} / \mathrm{s})$ & $H(\mathrm{~cm})$ & $B(\mathrm{~cm})$ & $B_{w}(\mathrm{~cm})$ & $L_{w}(\mathrm{~cm} / \mathrm{s})$ & $F r$ & $\operatorname{Re}$ & $S$ \\
\hline & & & & & & & $-1 / 6$ \\
\cline { 5 - 7 } & & & & & & & \\
\cline { 4 - 7 } & 5.3 & 30 & 10 & 30 & 0.17 & 6360 & 0 \\
\cline { 3 - 7 } & & & & & & & $1 / 15$ \\
\hline
\end{tabular}

Table2 Coefficient of determination for linear and quadratic approximations

\begin{tabular}{|c|c|c|}
\hline path distance (cm) & linear & quadratic \\
\hline 2 & 0.995 & 0.995 \\
\hline 5 & 0.989 & 0.993 \\
\hline 9 & 0.989 & 0.998 \\
\hline
\end{tabular}

